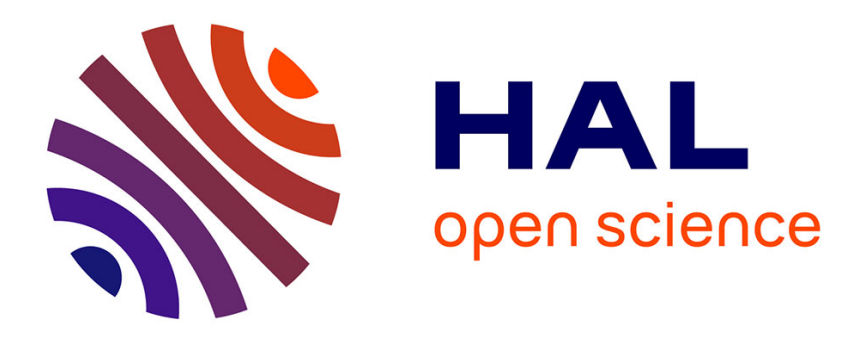

\title{
L'insertion professionnelle des jeunes et mesures publiques : des trajectoires différenciées entre hommes et femmes
}

Nathalie Havet

\section{- To cite this version:}

Nathalie Havet. L'insertion professionnelle des jeunes et mesures publiques: des trajectoires différenciées entre hommes et femmes. Annales d'Economie et de Statistique, 2006, n 81, pp. 225-250. halshs-00371238

\section{HAL Id: halshs-00371238 \\ https://shs.hal.science/halshs-00371238}

Submitted on 27 Mar 2009

HAL is a multi-disciplinary open access archive for the deposit and dissemination of scientific research documents, whether they are published or not. The documents may come from teaching and research institutions in France or abroad, or from public or private research centers.
L'archive ouverte pluridisciplinaire HAL, est destinée au dépôt et à la diffusion de documents scientifiques de niveau recherche, publiés ou non, émanant des établissements d'enseignement et de recherche français ou étrangers, des laboratoires publics ou privés. 


\title{
L'insertion professionnelle des jeunes et mesures publiques : des trajectoires différenciées entre hommes et femmes
}

\author{
Nathalie HAVET* \\ LÉO, Université d'Orléans (France) \\ Groupe d'Analyse Economique (Montréal, Canada)
}

${ }^{*}$ L'auteur tient à remercier, pour leurs précieuses remarques et suggestions, Guy Lacroix, Bruno Crépon et les deux rapporteurs anonymes de la revue ainsi que les participants aux Journées de l'AFSE (Rennes, 2004), au congrès de la SCSE (Mai, 2003) et aux Journées du Cirpée. L'auteur remercie aussi le Cirpée pour son hospitalité et sa mise à disposition de matériel nécessaire aux estimations de cet article. 


\section{Introduction}

La politique de l'emploi en France s'est progressivement développée depuis le début des années soixante-dix, au fur et à mesure de l'accroissement du chômage. D'abord centrée sur des mesures d'indemnisation et de placement, elle s'est ensuite engagée non seulement dans la création de stages et de contrats aidés, mais aussi dans des programmes de formation et de subventions à l'emploi sous la forme d'exonérations de cotisations sociales. Au cours des années quatre-vingt et quatre-vingt-dix, ces mesures publiques se sont mises en place à un rythme accéléré et se sont recentrées sur des publics cibles, au taux de chômage élevés : les jeunes et les chômeurs de longue durée ${ }^{1}$.

L'objet de cet article est d'évaluer l'efficacité des mesures publiques d'insertion en faveur des jeunes, en centrant plus spécifiquement l'analyse sur les différences entre sexes. L'efficacité est ici mesurée par l'effet du passage en contrat aidé ou programme gouvernemental de formation professionnelle sur les transitions vers un emploi stable. Cette problématique a déjà donné lieu à plusieurs travaux sur données françaises [Bonnal, Fougère \& Sérandon (1997), Brodaty, Crépon \& Fougère (2001), Magnac $\left.(1997,2000)^{2}\right]$. Toutefois, cette étude comparera l'influence des mesures publiques d'insertion sur les trajectoires des hommes et des femmes. L'idée sous-jacente est de savoir si ces dispositifs jouent le même rôle pour les deux sexes et s'ils pourraient être utilisés pour réduire les inégalités professionnelles.

L'évaluation quantitative est basée sur l'enquête Jeunes et Carrières 1997 , un complément de l'Enquête Emploi 1997 qui retrace les historiques familiaux et professionnels au niveau individuel. La nature des données a déterminé la méthode d'estimation utilisée et a imposé l'étude de l'impact des mesures publiques dans leur ensemble sans possibilité de les distinguer entre elles.

En l'absence d'expériences contrôlées, comme celles menées aux États-Unis [Garfinkel \& Manski (1992), Lalonde $(1995)^{3}$ ], l'évaluation statistique des mesures d'insertion repose sur l'utilisation de données longitudinales microéconomiques retraçant les histoires individuelles sur le marché du travail et s'appuie sur la modélisation des transitions des jeunes entre différents états ${ }^{4}$. Cependant, dans la littérature économétrique récente, la manière de modéliser les transitions n'est pas unique. D'une part, on trouve les modèles de transition multi-états multi-épisodes qui permettent de prédire simultanément les durées et les états de destination des transitions [Gritz (1993), Mealli, Pudney \& Thomas (1996), Bonnal et al. (1997), Gilbert, Kamionka \& Lacroix (2001)]. L'estimation de ces modèles fondés sur des processus de transition en temps continu nécessite des renseignements précis sur tous les états occupés successivement et sur les durées exactes de chaque séquence. D'autre part, on trouve des modèles à choix discrets de type logits polytomiques avec hétérogénéité inobservée [Card \& Sullivan (1988), Magnac (1997, 2000b), Jones \& Riddell (1999), Card \& Hyslop (2002)], qui estiment les probabilités de transitions entre deux dates distinctes dans le cadre d'une chaîne de Markov en temps discret.

\footnotetext{
${ }^{1}$ Pour une description de ces mesures publiques, voir DARES (1996).

${ }^{2}$ voir Fougère, Kramarz \& Magnac (2000) pour une comparaison des résultats de ces articles.

${ }^{3}$ Voir aussi Fougère (1998) pour un survol des expériences anglo-saxonnes et nord-européennes.

${ }^{4}$ Heckman, Ichimura \& Smith (1998) montrent que les évaluations fournies à partir de données non expérimentales peuvent être robustes. Voir aussi Magnac (2000a) pour une comparaison des méthodes expérimentale et économétrique.
} 
Or, dans l'Enquête Jeunes et Carrières 1997, les historiques ont été complétés rétrospectivement et donc les durées précises de chaque épisode des parcours individuels sont inconnues : seule la situation principale de chaque individu a été reportée pour chaque année. La méthode adaptée à l'étude de transitions à partir d'observations faites en temps discret a donc été privilégiée. Par rapport aux travaux antérieurs de ce courant, l'originalité de cette étude est d'estimer, par maximum de vraisemblance simulé, un logit polytomique dynamique à effets aléatoires individuels corrélés. Par exemple, sur données françaises, Magnac (1997, $2000 b$ ) a retenu une spécification à effets fixes et utilise des méthodes de vraisemblance conditionnelle ${ }^{5}$ pour traiter l'hétérogénéité inobservable corrélée. L'avantage de notre modélisation à effets aléatoires est qu'elle permet de quantifier d'une part l'impact des caractéristiques familiales et socio-économiques observables des individus sur les probabilités d'occupation des différents états du marché du travail et d'autre part les éventuelles corrélations entre caractéristiques inobservables spécifiques à chaque état. Les paramètres de transitions et l'impact des déterminants individuels, familiaux et conjoncturels, qu'ils varient ou non dans le temps, sont alors estimés simultanément ce qui n'est pas possible avec la méthode du maximum de vraisemblance conditionnelle. En revanche, la modélisation à effets fixes ne nécessite pas de spécifier la distribution des effets individuels : leurs estimations sont ainsi moins précises mais plus robustes car plus fiables que toute estimation avec une mauvaise spécification pour la loi des effets individuels. Cette étude permettra donc de montrer dans quelle mesure la prise en compte d'effets individuels aléatoires et corrélés sur des données plus récentes où l'observation des trajectoires est plus longue (jusqu'à 13 ans) modifie ou confirme les résultats déjà connus sur le sujet.

Compte tenu de la source de données utilisée, il n'est pas possible de distinguer les différentes mesures publiques d'insertion en faveur des jeunes puisque l'Enquête Jeunes et Carrières $199^{7}$ les agrège en une seule catégorie. C'est une limite en soi car plusieurs études antérieures [Bonnal et al. (1997) ou Brodaty et al. (2001)] ont mis en évidence la variabilité des impacts en fonction du type de mesure considérée et des caractéristiques des dispositifs mis en œuvre (en terme de formation, d'accompagnement, de secteur d'activité : marchand versus non marchand). Elle peut paraître d'autant plus importante dans l'analyse des différences entre sexes que les femmes se voient beaucoup plus souvent proposer des emplois subventionnés dans le secteur non marchand. Au contraire, les hommes profitent davantage des contrats d'apprentissage ou de formation professionnelle dans le secteur privé. C'est pourquoi, seul le rôle des mesures publiques d'une part sur les trajectoires professionnelles masculines et d'autre part sur les trajectoires féminines sera analysé, tout en gardant en tête lors des comparaisons entre sexes que la nature des dispositifs proposés peut être à l'origine des éventuels différentiels observés. Cet article permet néanmoins de savoir s'il existe des différences dans l'efficacité des mesures publiques d'insertion pour les hommes et les femmes, quelles soient liés aux contrats proposés, à de la discrimination, etc.

Dans la modélisation retenue, la situation professionnelle de l'individu est expliquée en fonction de ses caractéristiques individuelles et de son parcours antérieur sur le marché du travail, tout en tenant compte de la distribution des caractéristiques non observables. On peut ainsi identifier les facteurs les plus pénalisants en termes d'insertion. En particulier, on peut savoir si ce sont des caractéristiques individuelles prédéterminées (diplôme, environnement familial, etc) qui expliquent principalement les difficultés d'insertion ou si c'est le passé proche

\footnotetext{
${ }^{5}$ Pour ces techniques, voir Andersen (1973) et Chamberlain (1984).
} 
de l'individu, comme par exemple la répétition d'épisodes de chômage et d'emplois précaires, qui agit comme un mauvais signal auprès des employeurs. Un des résultats les plus marquants obtenus sur les données françaises de l'Enquête Jeunes et Carrières 1997 est que les facteurs handicapant l'insertion des jeunes diffèrent entre sexes : pour les hommes, la dépendance d'état, c'est-à-dire l'impact du passé de l'individu, serait prédominant alors que parmi les femmes, les difficultés d'insertion seraient plutôt à relier à un manque de qualifications ou à des événements familiaux (mise en couple, naissance d'enfants) qu'à leur passé professionnel.

La suite de cet article est organisé comme suit. La section 2 présente les données de l' Enquête Jeunes et Carrières 199\%. La section 3 détaille la méthode économétrique choisie pour modéliser les trajectoires des jeunes sur le marché du travail. Enfin, les résultats des estimations seront commentés dans la section 4.

\section{Les données}

L'aspect longitudinal de l'Enquête Jeunes et Carrières 1997, un complément de l'Enquête Emploi 1997 de l'INSEE, permet de suivre les trajectoires d'insertion des jeunes sur le marché du travail durant une assez longue période (jusqu'à 13 ans). Sont définis comme « Jeunes» dans l'enquête les moins de 30 ans ou les plus de 30 ans sortis depuis moins de sept ans du système éducatif en 1997. Pour chacun d'eux, on dispose d'un calendrier rétrospectif retraçant, sur une base annuelle, à la fois leurs historiques familiaux et professionnels depuis l'âge de 16 ans. On peut y repérer les mises en couple, les mariages, les séparations, les naissances d'enfants et la « situation principale » de l'individu vis-à-vis du marché du travail (études, emploi, chômage, inactivité). Dans le questionnaire, on entend par situation principale la période la plus longue ou celle qu'a occupé le plus de temps l'individu dans l'année (en cumulant éventuellement des périodes différentes). Par ailleurs, l'Enquête Emploi fournit des informations précises sur les caractéristiques de l'individu et de la famille d'origine pour 1997 (diplômes, éducation des parents, nombre de frères et sœurs, etc).

Lors de cette analyse, seulement six états sur le marché du travail seront distingués et non dix comme dans l'enquête initiale afin de considérer pour les estimations des populations qui restent suffisamment nombreuses dans chaque état. Ainsi, l'agrégation est la suivante :

1. Études : Élève, étudiant, stagiaire non rémunéré (1),

2. Mesures publiques d'insertion : Stagiaires de la formation professionnelle, contrat d'aide à l'emploi (3),

3. Emploi régulier : Indépendant (4), CDI complet (6), CDI partiel (7),

4. Emploi précaire : CDD, intérim, contrat saisonnier (5), apprentissage (2),

5. Chômage : Chômage (8),

6. Inactivité : Service National, Inactivité $(9,10)$.

Cette distinction est très proche voire similaire à celles utilisées par Magnac (1997, 2000), Bonnal, Fougère \& Sérandon $(1994,1997)$ et Cases \& Lollivier (1994), dans leurs analyses du chômage et des transitions sur le marché du travail en France. Deux types d'emplois, les emplois réguliers et les emplois précaires sont distingués afin de tenir compte du dualisme du marché du travail [Piore (1978)]. 
En outre, ne sont gardés dans l' échantillon que les individus qui avaient définitivement fini leurs études ${ }^{6}$ et dont les trajectoires professionnelles ne comportaient ni trous ni incohérence. Finalement, l'échantillon final est constitué de 3585 jeunes (1 712 hommes et 1873 femmes) qui ont été repérés dans 20250 états, décrits au tableau 1. Il est à noter que le nombre de situations (ou transitions) observées varie d'un individu à l'autre en fonction de leur âge et de leur date de fins d'études. Pour ceux qui sont sortis le plus récemment du système scolaire, on observe une seule transition et pour les plus vieux au plus treize transitions. Le tableau 2 présente quelques statistiques descriptives de notre échantillon en distinguant les hommes et les femmes. Les variables explicatives variant dans le temps sont l'âge des jeunes, le nombre d'enfants, la situation matrimoniale et le fait que les parents soient divorcés ou non. Les autres variables sont considérées comme fixes dans le temps car on ne dispose de ces renseignements que pour l'année 1997.

\begin{tabular}{|l|ccccc|c|}
\hline $\mathbf{k}$ & & & & & & \\
$\mathbf{j}$ & Mesures pub. & CDI & CDD & Chômage & Inactivité & Total \\
\hline Etudes & 319 & 748 & 643 & 580 & 228 & 2518 \\
Mesures pub. & $\mathbf{5 5 2}$ & 315 & 268 & 286 & 91 & 1512 \\
CDI & 44 & $\mathbf{7 3 1 6}$ & 155 & 285 & 182 & 7982 \\
CDD & 88 & 587 & $\mathbf{2 0 3 2}$ & 375 & 168 & 3250 \\
Chômage & 183 & 328 & 370 & $\mathbf{1 3 9 2}$ & 112 & 2385 \\
Inactivité & 69 & 494 & 306 & 243 & $\mathbf{1 4 9 1}$ & 2603 \\
\hline Total & 1255 & 9788 & 3774 & 3161 & 2272 & $\mathbf{2 0 2 5 0}$ \\
\hline
\end{tabular}

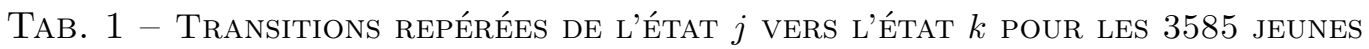

Les déclarations des statuts occupés par les jeunes sur le marché du travail, depuis leur sortie du système éducatif jusqu'en 1997, permettent de décrire les transitions d'une année sur l'autre. Le tableau 3 reporte les probabilités d'être dans les différents états $(k)$ une année, conditionnellement à l'état occupé l'année précédente $(j)$ et ce en distinguant par sexes.

En italique apparaissent des indicateurs de récurrence des états ou en d'autres termes les probabilités conditionnelles d'avoir la même situation principale durant deux années consécutives. Le statut le plus récurrent correspond aux contrats à durée indéterminée (92\%). Cela signifie qu'une fois que les jeunes ont trouvé un emploi régulier, ils ont tendance à rester dans cet emploi ou à ne le quitter que pour un emploi de même nature. En revanche, les mesures publiques d'insertion telles que les formations et les stages rémunérés sont beaucoup plus temporaires (taux de récurrence de 37\%) dans le parcours des jeunes.

Les autres chiffres du tableau 3 décrivent les véritables transitions entre statuts. Ils montrent que l'insertion professionnelle est difficile. L'accès à un emploi régulier est incertain, tellement la précarité est répandue chez les jeunes. Deux tiers des sortants du système éducatif voient leur entrée dans la vie active se solder par un épisode de chômage, un emploi temporaire ou une mesure publique d'insertion. Plus précisément, les jeunes confrontés au chômage semblent avoir moins de chances d'obtenir un emploi stable que ceux qui ont pu

\footnotetext{
${ }^{6}$ Très peu de jeunes font un retour aux études après être sortis une première fois du système éducatif (moins d'un 1\% des transitions). C'est pourquoi, toujours par souci d'avoir des groupes à effectifs suffisamment nombreux lors de l'estimation, ces individus reprenant leurs études sont exclus de l'échantillon.
} 


\begin{tabular}{|c|c|c|}
\hline & HOMMES & FEMMES \\
\hline Age moyen en 1997 & 25,34 & 25,43 \\
\hline En couple en 1997 & $41,6 \%$ & $59,2 \%$ \\
\hline \multicolumn{3}{|l|}{ Nbre d'enfants en 1997} \\
\hline 0 & $81,1 \%$ & $61,4 \%$ \\
\hline 1 & $13,3 \%$ & $23,6 \%$ \\
\hline 2 et plus & $5,6 \%$ & $15,0 \%$ \\
\hline Parents divorcés en 1997 & $18,0 \%$ & $19,9 \%$ \\
\hline \multicolumn{3}{|l|}{ Diplômes } \\
\hline sans diplôme, 1er cycle du secondaire & $30,3 \%$ & $30,5 \%$ \\
\hline enseignement professionnel court & $35,9 \%$ & $27,4 \%$ \\
\hline enseignement professionnel long & $11,5 \%$ & $12,0 \%$ \\
\hline Baccalauréat général & $5,1 \%$ & $10,1 \%$ \\
\hline Baccalaureat +2 ans & $11,1 \%$ & $12,1 \%$ \\
\hline Diplôme supérieur & $6,2 \%$ & $8,0 \%$ \\
\hline \multicolumn{3}{|l|}{ Nombre de frères et sœurs } \\
\hline 0 & $8,8 \%$ & $7,5 \%$ \\
\hline 1 & $29,1 \%$ & $28,2 \%$ \\
\hline 2 & $29,0 \%$ & $26,8 \%$ \\
\hline 3 et plus & $33,2 \%$ & $37,5 \%$ \\
\hline \multicolumn{3}{|l|}{ Niveau d'éducation du père } \\
\hline sans diplôme, enseignement primaire & $51,0 \%$ & $58,1 \%$ \\
\hline bepc, baccalauréat général & $10,6 \%$ & $10,8 \%$ \\
\hline enseignement professionnel court & $27,6 \%$ & $21,1 \%$ \\
\hline enseignement professionnel long & $3,2 \%$ & $2,6 \%$ \\
\hline Diplôme supérieur au bac & $7,7 \%$ & $7,3 \%$ \\
\hline \multicolumn{3}{|l|}{ Niveau d'éducation de la mère } \\
\hline sans diplôme, enseignement primaire & $54,0 \%$ & $57,0 \%$ \\
\hline bepc, baccalauréat général & $17,0 \%$ & $18,5 \%$ \\
\hline enseignement professionnel court & $20,56 \%$ & $16,9 \%$ \\
\hline enseignement professionnel long & $1,9 \%$ & $1,2 \%$ \\
\hline Diplôme supérieur au bac & $6,5 \%$ & $6,3 \%$ \\
\hline
\end{tabular}

TAB. 2 - Statistiques Descriptives

suivre un programme gouvernemental ou qui ont pu décrocher un contrat à durée déterminée. En effet, les chômeurs transitent davantage vers les emplois précaires que directement vers les emplois réguliers.

Néanmoins, l'emploi à durée indéterminée est l'état de destination principal des autres situations (mesure publique, CDD, inactivité) et les mesures publiques d'insertion semblent le meilleur tremplin pour y accéder, au moins pour les hommes. Les transitions entre états temporaires (chômage, mesure publique, CDD) sont aussi importantes. 18\% des individus en mesures publiques d'insertion sont en emploi précaire ou au chômage l'année suivante, $12 \%$ des jeunes en contrat à durée déterminée se retrouvent au chômage un an après et les jeunes chômeurs transitent surtout vers des CDD. 


\begin{tabular}{|l|ccccc|}
\hline \multicolumn{1}{|c|}{$\mathbf{k}$} & \multicolumn{5}{c|}{ HoMmES } \\
$\mathbf{j}$ & Mesures pub. & CDI & CDD & Chômage & Inactivité \\
\hline Etudes & 13,7 & 32,6 & 26,4 & 23,1 & 4,2 \\
Mesures pub. & 34,6 & 26,0 & 16,4 & 16,5 & 6,5 \\
CDI & 0,5 & 92,0 & 2,1 & 3,1 & 2,6 \\
CDD & 2,8 & 19,1 & 63,3 & 8,8 & 6,1 \\
Chômage & 15,9 & 15,5 & 17,5 & 54,0 & 6,3 \\
Inactivité & 4,6 & 40,4 & 25,0 & 18,4 & 11,7 \\
\hline \hline \multicolumn{1}{|c}{$\mathbf{k}$} & & FEMMES & & \\
$\mathbf{j}$ & Mesures pub. & CDI & CDD & Chômage & Inactivité \\
\hline Etudes & 12,1 & 28,3 & 25,1 & 23,0 & 11,4 \\
Mesures pub. & 38,1 & 16,4 & 18,9 & 21,0 & 5,6 \\
CDI & 0,7 & 91,5 & 1,7 & 4,1 & 1,9 \\
CDD & 2,6 & 17,1 & 61,8 & 14,1 & 4,4 \\
Chômage & 8,5 & 12,5 & 14,3 & 61,0 & 9,8 \\
Inactivité & 1,4 & 4,5 & 2,7 & 3,2 & 88,3 \\
\hline
\end{tabular}

TAB. 3 - Probabilités de transitions de L'État $j$ Vers L'État $k$ (EN \%)

La différence la plus flagrante entre sexes concerne leurs comportements vis-à-vis de l'inactivité. A la fin de leurs études, une part non-négligeable de femmes (11,5\%) décident de ne pas participer au marché du travail. Chez elles, l'inactivité est un état absorbant : une fois à l'écart du travail rémunéré, il est rare qu'elles y reviennent. Au contraire, chez les hommes, les périodes d'inactivité sont temporaires et sans doute de courte durée. 65,5\% des hommes inactifs retrouvent un emploi un an après. Ces différences tiennent à la nature même de l'inactivité. Pour les hommes, c'est essentiellement le service national qui les oblige à se retirer temporairement du marché du travail. Cela explique pourquoi davantage d'hommes que de femmes en emplois ou mesures publiques d'insertion deviennent inactifs. Chez les femmes, l'inactivité représente davantage un choix de vie privilégiant la famille. Mais pour certaines, l'inactivité est le reflet de leur échec d'insertion professionnelle. A la suite d'une période de chômage prolongée, certaines femmes par découragement se retirent du marché du travail. Elles sont plus nombreuses à transiter du chômage vers l'inactivité que les hommes (10\% contre $6 \%$ ).

Il faut dire que l'insertion reste plus difficile pour les femmes. A la sortie du système éducatif, elles obtiennent moins d'emplois réguliers. Par ailleurs, quand elles arrivent à obtenir une formation rémunérée, un contrat à durée déterminée ou indéterminée, elles ont une probabilité plus forte de se retrouver au chômage. En particulier, $14 \%$ de femmes contre $9 \%$ des hommes en emplois précaires une année sont confrontés au chômage l'année suivante. Quel que soit l'état considéré, les femmes ont plus de difficultés à être embauchées sous un contrat à durée indéterminée. Les emplois temporaires, mais surtout les mesures publiques d'insertion ne semblent pas d'aussi bons tremplins vers les emplois réguliers pour les femmes que pour les hommes.

Toutefois, ces comparaisons sont à prendre avec précaution car nos deux groupes de référence n'ont pas tout à fait les mêmes caractéristiques individuelles et familiales, voire les mêmes préférences vis-à-vis du marché du travail. D'où la nécessité de recourir à des modélisations économétriques des transitions qui nous permettront en plus d'analyser l'in- 
fluence de certaines variables tels que le diplôme ou la présence d'enfants sur le processus d'insertion professionnelle.

\section{Modélisations économétriques des transitions sur le marché du travail}

\subsection{Spécification économétrique}

Pour modéliser les parcours professionnels des jeunes, un logit polytomique dynamique avec effets aléatoires corrélés est utilisé. Cette spécification reprend le cadre des modèles à variables latentes à la McFadden (1974). Mais on introduit les variables décrivant les états occupés à la date précédente comme variables explicatives, ce qui permet une description dynamique des trajectoires en temps discret sans recourir à un processus en temps continu. Enfin, en spécifiant des effets individuels aléatoires, on distingue ce qui vient des goûts et des qualités inobservables des jeunes et qui les ont conduit dans les états qu'ils occupent (hétérogénéité inobservable), de ce qui vient de l'avantage que leur confère l'occupation de certains états pour l'accession à d'autres états. Cette méthode permet ainsi de séparer la dépendance d'état et l'hétérogénéité inobservable corrélée, selon la terminologie de Heckman (1981a, 1981b). Regardons plus en détails la structure générale de ce modèle.

A chaque date $t$, la variable latente $y_{i t l}^{*}$ décrit la propension de l'individu $i$ d'être dans l'état $l$ du marché du travail (Mesure publique d'insertion, Chômage, CDD, CDI, Inactivité). Elle s'écrit en fonction de variables socio-démographiques $X_{i t}$ (âge, diplôme, sexe, etc), de l'état occupé l'année précédente $y_{i(t-1) k}$ et de termes d'hétérogénéité inobservable entre les individus $\varepsilon_{i l t}$ :

$$
y_{i t l}^{*}=\beta_{l}^{\prime} X_{i t}+\sum_{k=0}^{L} \delta_{k l} y_{i(t-1) k}+\varepsilon_{i l t} .
$$

Ces propensions captent à la fois ce qui relève des préférences des individus envers les états et ce qui relève des contraintes d'accès aux états qu'ils subissent comme par exemple les phénomènes de discrimination pour les femmes. L'état observé, $y_{i t}$, est supposé être celui qui correspond à la propension maximale : $y_{i t}=l$ si $y_{i t l}^{*}=\max _{k}\left(y_{i t k}^{*}\right)$.

Pour décrire la dynamique de l'insertion sur le marché du travail, il est indispensable de tenir compte de l'itinéraire passé des individus. Par exemple, il est intuitif de penser qu'un individu aura moins de chances de se retrouver à la date $t$ dans un emploi régulier si son historique professionnel est davantage composé d'épisodes de chômage que d'épisodes d'emploi. Or la démarche la plus usuelle ${ }^{7}$ pour décrire les transitions est de supposer que celles-ci peuvent être représentées par une chaîne de Markov d'ordre 1. Par là-même, on fait l'hypothèse que la loi de probabilité $y_{i t}$, à un instant quelconque, conditionnellement à un résumé fini de son histoire $y_{i(t-1)}, y_{i(t-2)}, y_{i(t-3)}, \ldots, y_{i(t-p)}$ ne dépend que de sa réalisation la plus proche dans cet historique $y_{i(t-1)}$. En d'autres termes, on considère que la probabilité d'occuper un état $l$ à la date $t$ est complètement caractérisée par la probabilité conditionnelle $P\left(y_{i t}=l \mid y_{i(t-1)}=k\right)$. C'est pourquoi, on inclut ici dans la liste des variables explicatives les états occupés l'année précédente.

\footnotetext{
${ }^{7}$ voir Card \& Sullivan (1988), Magnac (1997), Jones \& Riddell (1999), Card \& Hyslop (2002).
} 
Les coefficients $\delta_{k l}$ mesurent l'effet de l'état $k$ occupé précédemment sur la propension à entrer dans l'état $l$. Toutefois, pour en obtenir une estimation non-biaisée, il faut tenir compte de l'hétérogénéité individuelle inobservée, ce qui est fait ici via l'introduction d'effets aléatoires individuels spécifiques à chaque état. Plus précisément, on considère que les termes d'hétérogénéité inobservable $\varepsilon_{i l t}$ se décomposent en deux éléments :

$$
\varepsilon_{i t l}=\alpha_{i l}+u_{i t l}
$$

avec $\alpha_{i l}$ un terme reflétant les effets individuels spécifiques à chaque état.

Si on se contentait juste d'introduire les variables associées à l'état passé, les coefficients $\delta_{k l}$ capteraient certes ce qui vient de l'avantage ou du désavantage que leur confère l'occupation de certains états pour l'accession à d'autres états, mais aussi les goûts et qualités inobservables des jeunes qui les ont conduit dans ces états. On tendrait alors à biaiser à la hausse les coefficients associés aux états passés. La modélisation explicite des effets individuels $\alpha_{i l}$ permet de faire la distinction entre la véritable dépendance d'état et l'hétérogénéité inobservable.

Par ailleurs, l'état de départ de tous les individus de notre échantillon est identique : ils sont tous en études. Toutefois, cet état ne peut sans doute pas être considéré comme exogène puisque les jeunes ne l'atteignent pas tous au même âge. Pour en tenir compte, cela nous impose de spécifier la distribution de l'hétérogénéité inobservable conditionnellement à cet état de sortie des études, $y_{i 0}{ }^{8}$ [Wooldridge (2002)].

Sous l'hypothèse que les termes d'erreurs $u_{i t l}$ suivent des lois à valeurs extrêmes de type I, indépendantes au cours du temps et entre états, la probabilité inconditionnelle que l'individu $i$ occupe l'état $l$ à la date $t$ a pour expression :

$$
P\left(y_{i t}=l \mid y_{i(t-1)}=k, X_{i t}, \alpha_{i l}\right)=\frac{\exp \left(\beta_{l}^{\prime} X_{i t}+\sum_{h=0}^{L} \delta_{h l} y_{i(t-1) h}+\alpha_{i l}\right)}{\sum_{k} \exp \left(\beta_{k}^{\prime} X_{i t}+\sum_{h=0}^{L} \delta_{h l} y_{i(t-1) h}+\alpha_{i k}\right)} .
$$

Comme dans tout logit multinomial, seuls les coefficients $\beta_{j}-\beta_{0}, \delta_{k l}-\delta_{k 0}$ et $\alpha_{i l}-\alpha_{i 0}$ sont identifiables, donc on normalise à zéro les vecteurs des paramètres $\left(\beta_{0}, \delta_{k 0}, \alpha_{i 0}\right)$ correspondant à l'état de référence, soit l'inactivité. L'inactivité est ainsi la référence non seulement pour les états de destination mais aussi pour les états d'origine introduits comme variables explicatives. Les coefficients estimés $\delta_{h l}$ s'interprètent donc comme des écarts par rapport à l'impact d'un épisode antérieur d'inactivité sur une transition vers un nouvel épisode d'inactivité.

L'originalité ici par rapport aux modèles à choix discrets de la littérature décrivant la dynamique des trajectoires professionnelles est de considérer que les effets aléatoires individuels sont corrélés entre eux ${ }^{9}$. On suppose que les effets individuels suivent une distribution normale $^{10}$ multivariée d'ordre $4:\left(\alpha_{i 1}, \alpha_{i 2}, \alpha_{i 3}, \alpha_{i 4} \mid y_{i 0}\right)^{\prime} \sim \mathcal{N}(a, \Omega)$ avec $\Omega$ de forme générale, c'est-à-dire avec la possibilité de corrélations non-nulles entre les différents aléas. Cette hypothèse est importante car un individu qui a une forte préférence pour les contrats à durée

\footnotetext{
${ }^{8}$ L'échantillon est lui-même observé conditionnellement au fait d'avoir terminé sa scolarité. Cela revient à traiter la sortie du système scolaire comme endogène.

${ }^{9}$ En revanche, les modèles de transitions multi-états multi-épisodes tiennent eux déjà compte d'effets individuels corrélés, entre états de destination et parfois aussi entre états d'origine. Voir à ce propos Gilbert et al. (2001).

${ }^{10}$ Dans les estimations paramétriques, le choix de la loi normale pour caractériser l'hétérogénéité inobservable est usuel. Voir Card \& Hyslop (2002) et Magnac (2000b).
} 
indéterminée aura sans doute aussi un goût marqué pour les autres formes d'emplois par rapport à l'inactivité, traduisant le fait qu'il est décidé à participer au marché du travail. On s'attend ainsi à des corrélations positives et assez élevées entre les effets individuels. Ici, on a $\operatorname{cov}\left(\varepsilon_{i l t}, \varepsilon_{i k t}\right)=\mathbb{E}\left[\left(\alpha_{i l}+u_{i l t}\right)\left(\alpha_{i k}+u_{i k t}\right)\right]=\sigma_{\alpha_{l}, \alpha_{k}}^{2}$. Les composantes inobservées des propensions associées aux états $l$ et $k$ sont reliées entre elles par la corrélation des termes d'hétérogénéité individuelle et donc la propriété IIA très restrictive est contournée. En permettant une plus grande flexibilité dans la structure des corrélations des termes d'erreurs, la spécification avec des effets aléatoires conduit à des estimateurs plus efficaces.

Enfin, on doit tenir compte des choix répétés des individus qui peuvent être une source de biais dans l'estimation. Cela nécessite de raisonner sur les trajectoires, c'est-à-dire sur l'ensemble des états successifs occupés par individu. La probabilité conditionnelle d'observer la succession d'états $\left(y_{i 1}, \ldots, y_{i T_{i}} \mid y_{i 0}\right)$ pour l'individu $i$ est simplement le produit des probabilités décrites à l'équation (3) :

$$
S\left(y_{i 1}, \ldots, y_{i T_{i}} \mid \alpha_{i l}, y_{i 0}\right)=\prod_{t}^{T_{i}} P\left(y_{i t}=l \mid y_{i(t-1)}=k, X_{i t}, \alpha_{i l}\right) .
$$

Il est à noter que la période d'observation $T_{i}$ varie entre les individus en fonction de leur âge et de leur date de fin d'études : $T_{i}$ est compris entre 2 et 14 .

Or comme les effets individuels ne sont pas observables, on ne peut identifier les $\beta$ et $\delta$. On doit intégrer la probabilité conditionnelle sur toutes les valeurs possibles de $\alpha_{i l}$ pour obtenir la probabilité inconditionnelle que l'individu $i$ ait la trajectoire $\left(y_{i 1}, \ldots, y_{i T_{i}}\right)$. Elle s'écrit :

$$
S\left(y_{i 1}, \ldots, y_{i T_{i}}\right)=\iiint \int S\left(y_{i 1}, \ldots, y_{i T_{i}} \mid \alpha_{i l}, y_{i 0}\right) . f\left(\alpha_{i} \mid a, \Omega, y_{i 0}\right) d \alpha_{i 1} d \alpha_{i 2} d \alpha_{i 3} d \alpha_{i 4}
$$

et la vraisemblance individuelle inconditionnelle :

$\mathcal{L}_{i}=\iiint \int \prod_{t} \prod_{l}\left[\frac{\exp \left(\beta_{l}^{\prime} X_{i t}+\sum_{h=0}^{L} \delta_{h l} y_{i(t-1) h}+\alpha_{i l}\right)}{\sum_{k} \exp \left(\beta_{k}^{\prime} X_{i t}+\sum_{h=0}^{L} \delta_{h l} y_{i(t-1) h}+\alpha_{i k}\right)}\right]^{y_{i t l}} f\left(\alpha_{i} \mid a, \Omega, y_{i 0}\right) \cdot d \alpha_{i 1} d \alpha_{i 2} d \alpha_{i 3} d \alpha_{i 4}$,

avec $y_{i t l}=1$ si $y_{i t}=l$ et $y_{i t l}=0$ sinon.

Au final, la log-vraisemblance à maximiser pour estimer les paramètres $\beta$ associés aux variables explicatives et les paramètres de la distribution des effets individuels $a$ et $\Omega$, est égale à la somme des log-vraisemblances individuelles, soit $\ln \mathcal{L}=\sum_{i} \ln \mathcal{L}_{i}$. Or l'estimation exacte des paramètres par la méthode du maximum de vraisemblance n'est en pratique pas possible car l'intégrale de l'équation (6) ne peut pas être calculée analytiquement. En outre, l'intégration numérique par la technique bien connue de quadrature gaussienne n'est pas ici réalisable en raison des temps de calculs associés aux intégrales de dimension supérieures ou égales à trois. En conséquence, on a eu recours à une procédure de simulations pour obtenir une approximation de cette intégrale et nos estimateurs sont les solutions de la maximisation de la log-vraisemblance simulée.

\subsection{Méthode d'estimation : Maximum de vraisemblance simulé}

L'idée de base des techniques de simulations est de remplacer l'intégrale multi-dimensionnelle dans l'expression des estimateurs théoriques exacts par des simulateurs de probabilités facile- 
ment calculables. Lerman \& Manski (1981) ont introduit ce concept et présenté une procédure d'approximation relativement simple, basée sur les fréquences mais dont les propriétés asymptotiques étaient assez pauvres. D'autres travaux ont ensuite proposé des simulateurs ("smooth simulators") comblant cette lacune. Ils incluent notamment les articles de McFadden (1989) et Pakes \& Pollard (1989) pour la méthode des moments simulés et ceux de Gouriéroux \& Monfort (1996) et Laroque \& Salanié (1993) sur le pseudo-maximum de vraisemblance simulé. Ici, on utilise cette dernière démarche car elle possède de nombreux avantages : elles est assez facile à mettre en œuvre car elle se fonde sur un critère d'optimisation très proche du maximum de vraisemblance classique ; elle est significativement plus rapide en termes de calculs que celles des moments simulés [Stern (1997)] et sa fonction objectif se comporte mieux; et quand le nombre de simulations est important, les estimateurs obtenus sont efficaces et ont les propriétés statistiques bien connues du maximum de vraisemblance [Hajivassiliou \& McFadden (1998)]

Formellement, la probabilité $S\left(y_{i 1}, \ldots, y_{i T_{i}}\right)$ de l'équation (5) est approximée par la somme de probabilités conditionnelles évaluées pour des valeurs particulières de $\alpha_{i l}$. Pour chaque individu, on fait $R$ tirages de $\left(\alpha_{i 1}^{r}, \alpha_{i 2}^{r}, \alpha_{i 3}^{r}, \alpha_{i 4}^{r}\right)^{\prime}$ à partir de la distribution $\mathcal{N}(a, \Omega)$ et on calcule la probabilité conditionnelle $S\left(y_{i 1}, \ldots, y_{i T_{i}} \mid \alpha_{i l}^{r}\right)$ pour chaque ensemble de valeurs. La moyenne des $R$ probabilités obtenues est alors un simulateur sans biais de la probabilité inconditionnelle :

$$
\begin{aligned}
\tilde{S}\left(y_{i 1}, \ldots, y_{i T_{i}}\right) & =\frac{1}{R} \sum_{r=1}^{R} S\left(y_{i 1}, \ldots, y_{i T_{i}} \mid \alpha_{i l}^{r}\right) \\
& =\frac{1}{R} \sum_{r=1}^{R} \prod_{t}^{T_{i}} \prod_{l}\left[\frac{\exp \left(\beta_{l}^{\prime} X_{i t}+\sum_{h=0}^{L} \delta_{h l} y_{i(t-1) h}+\alpha_{i l}^{r}\right)}{\sum_{k} \exp \left(\beta_{k}^{\prime} X_{i t}+\sum_{h=0}^{L} \delta_{h l} y S_{i(t-1) h}+\alpha_{i k}^{r}\right)}\right]^{y_{i t l}},
\end{aligned}
$$

avec $\left(\alpha_{i 1}^{r}, \alpha_{i 2}^{r}, \alpha_{i 3}^{r}, \alpha_{i 4}^{r}\right)^{\prime}$ le $r$-ième tirage à partir de $f(. \mid a, \Omega)$ et $\tilde{S}\left(y_{i 1}, \ldots, y_{i T_{i}}\right)$ la probabilité simulée de la trajectoire de la personne $i$. La fonction de log-vraisemblance simulée est alors construite comme $: \tilde{\ln } \mathcal{L}=\sum_{i} \ln \tilde{S}\left(y_{i 1}, \ldots, y_{i T_{i}}\right)$ et les paramètres estimés sont ceux qui maximisent cette fonction. Ils sont asymptotiquement sans biais mais risquent d'être biaisés si le nombre de tirage est faible. De même, leur variance diminue quand le nombre de tirages $R$ augmente $^{11}$.

Dans la pratique, les tirages dans la distribution normale multivariée de moyenne $a$ et de matrice de variance-covariance $\Omega$ sont effectués à l'aide de la décomposition de Cholesky. Elle consiste à spécifier le vecteur $\alpha_{i}=\left(\alpha_{i 1}, \alpha_{i 2}, \alpha_{i 3}, \alpha_{i 4}\right)^{\prime}$ comme une combinaison linéaire de quatre variables indépendantes distribuées selon une loi normale standard univariée :

$$
\alpha_{i}=a+T \eta_{i} ; \eta_{i} \sim \mathcal{N}(0,1),
$$

où $T$ est une matrice triangulaire inférieure - dont les éléments sont appelés facteurs de Cholesky -, telle que $T T^{\prime}=\Omega$. Ainsi, la log-vraisemblance simulée est réécrite en remplaçant les $\alpha_{i}$ par leur expression (équation (8)) et ce sont les paramètres $a$ et $T$ qui sont directement estimés. Les éléments de la matrice $\Omega$ sont ensuite calculés en effectuant le produit $\hat{\Omega}=\hat{T} \hat{T}^{\prime}$ et les écarts-types de ces éléments sont obtenus par la méthode delta ${ }^{12}$.

\footnotetext{
${ }^{11}$ Voir McFadden \& Train (2000), Lee (1992).

${ }^{12}$ Cette méthode utilise le résultat général suivant : pour $\Psi=f(p), \operatorname{Var}(\Psi)=\left(\frac{\partial \Psi}{\partial p}\right)^{\prime} \operatorname{Var}(p)\left(\frac{\partial \Psi}{\partial p}\right)$ où $\Psi$ et
} 
Un grand nombre de tirages est nécessaire pour s'assurer que l'erreur de simulation dans les paramètres estimés reste faible. En effet, même si la probabilité simulée $\tilde{S}\left(y_{i 1}, \ldots, y_{i T_{i}}\right)$ est un estimateur sans biais de la vraie probabilité $S\left(y_{i 1}, \ldots, y_{i T_{i}}\right)$, la log-vraisemblance simulée n'est pas un estimateur sans biais de la véritable log-vraisemblance à cause de la non-linéarité de la fonction logarithme. La log-vraisemblance simulée sous-estime la vraie valeur de la logvraisemblance. Néanmoins ce biais diminue quand le nombre de tirages $R$ augmente. Ainsi, la précision des paramètres estimés par la méthode du maximum de vraisemblance simulé dépend de façon cruciale de l'utilisation d'un grand nombre de tirages. C'est pourquoi, on utilise 1000 tirages pour chaque individu $(R=1000)$ afin de limiter les biais ${ }^{13}$. Néanmoins, quand $R$ est fini, la matrice de variance-covariance est en générale sous-estimée [Newey \& McFadden (1994), McFadden \& Train (2000)]. Les tests de significativité seront donc fondés sur la matrice robuste de variance-covariance qui tient compte de l'erreur de simulation et qui a pour expression $H^{-1} G H^{-1}$ avec $H$ la vraie matrice hessienne des paramètres et $G$ le produit croisé des gradients, soit l'approximation par le BHHH de la hessienne. On va désormais présenter les résultats obtenus.

\section{Résultats}

En plus de l'historique professionnel, on a retenu comme variables explicatives des probabilités d'occupations des statuts non seulement un certain nombre de caractéristiques individuelles (âge, situation matrimoniale, nombre d'enfants, diplôme le plus élevé) et familiales (nombre de frères et de sœurs, parents divorcés, niveau d'éducation des parents) mais aussi des variables dichotomiques temporelles afin de capter les effets de conjoncture. Par ailleurs, est introduite la variable nombre d'enfants* ${ }^{*}$ exe où sexe $=1$ pour les femmes car au vu de la répartition des tâches au sein des ménages - entre $70 \%$ et $80 \%$ de la production domestique comprenant l'éducation des enfants est assumé par les femmes ${ }^{14}$, il est fort probable que la naissance d'enfants influence différemment les parcours professionnels des hommes et des femmes.

On commencera par mettre en évidence l'importance des corrélations existantes entre les préférences individuelles. Puis on analysera les déterminants individuels et familiaux des différents comportements vis-à-vis du marché du travail. Enfin, l'efficacité des politiques gouvernementales en faveur des jeunes sera évaluée.

\subsection{Dépendance d'état et hétérogénéité inobservable}

Afin de tester la légitimité de la prise en compte de l'hétérogénéité inobservée et des corrélations entre effets individuels de chaque état, deux cas particuliers du modèle général ont été estimés. Le premier postule que $\Omega=0$, ce qui revient à négliger l'hétérogénéité inobservée $^{15}$. Le deuxième considère les termes d'hétérogénéité individuels comme indépendants, hypothèse équivalente à $\Omega$ matrice diagonale. L'Annexe présente, pour ces trois modèles, les

$p$ sont des vecteurs. Dans notre cas, $p$ est un vecteur composé des éléments de $T, \Psi$ est un vecteur composé des éléments de $\Omega$ et $f$ est égale à $T T^{\prime}$.

${ }^{13}$ La norme dans ce type de travaux est souvent de 250 à 500 tirages.

${ }^{14}$ Chiffre trouvé par Glaude (1999) et Anxo, Flood \& Kocoglu (2002).

${ }^{15}$ Cette hypothèse est équivalente à la nullité des facteurs de Cholesky. 
paramètres des états passés et de la distribution de l'hétérogénéité inobservable ainsi que les coefficients associés aux déterminants individuels, familiaux et conjoncturels des probabilités d'occupation des statuts.

Dans le cas où $\Omega$ est une matrice diagonale, les écarts-types des termes d'hétérogénéité sont élevés et significatifs, ce qui signifie qu'il est primordial d'en tenir compte. D'ailleurs, on rejette formellement l'hypothèse $\Omega=0$ à l'aide d'un test du rapport de vraisemblance : la statistique du test effectué à partir des colonnes 1 et 2 vaut 497,18 pour une valeur critique $\chi_{.05}^{2}(4)$ de 9,49 . En outre, il ressort qu'omettre l'hétérogénéité inobservée tend à biaiser à la hausse les coefficients des états passés. Ce biais est dû à la "fausse dépendance d'état" [Heckman (1981a)]. Les populations dans chaque situation du marché du travail, à chaque période, sont sélectionnées sur la base de caractéristiques inobservées qui influencent de façon cruciale leurs trajectoires professionnelles futures. Par exemple, les individus qui ne sont pas embauchés en contrat à durée indéterminée à la période précédente ont des caractéristiques qui sont défavorables à leur présence dans cet état. Au contraire, les individus occupant ces emplois ont des caractéristiques qui favorisent leur maintien dans cet état. C'est pourquoi, si on ne tient pas compte de l'hétérogénéité individuelle, on sur-estime la dépendance d'état.

Le tableau 4 révèle l'importance des corrélations existantes entre les effets individuels de chaque état. Il reporte la matrice de variance-covariance estimée $\hat{\Omega}$ du modèle général, les t de Student associés à cette matrice et les corrélations entre les aléas.

\begin{tabular}{|c|c|c|c|c|}
\hline \multicolumn{5}{|c|}{$\begin{array}{c}\text { Matrice de variance-covariance : } \\
\hat{\Omega}=\hat{T} \hat{T}^{\prime}\end{array}$} \\
\hline & $\alpha_{i 1}$ & $\alpha_{i 2}$ & $\alpha_{i 3}$ & $\alpha_{i 4}$ \\
\hline$\alpha_{i 1}$ & 2,872 & 2,147 & 1,777 & 1,746 \\
\hline$\alpha_{i 2}$ & 2,147 & 2,992 & 1,898 & 1,717 \\
\hline$\alpha_{i 3}$ & 1,777 & 1,898 & 2,198 & 1,059 \\
\hline$\alpha_{i 4}$ & 1,746 & 1,717 & 1,059 & 2,380 \\
\hline \multicolumn{5}{|c|}{ t de Student des covariances } \\
\hline & 8,087 & 6,789 & 5,787 & 5,731 \\
\hline & 6,789 & 7,939 & 6,031 & 5,416 \\
\hline & 5,787 & 6,031 & 6,266 & 3,668 \\
\hline & 5,731 & 5,416 & 3,668 & 5,843 \\
\hline \multicolumn{5}{|c|}{ Matrice de corrélation } \\
\hline & 1,000 & 0,732 & 0,707 & 0,668 \\
\hline & 0,732 & 1,000 & 0,740 & 0,643 \\
\hline & 0,707 & 0,740 & 1,000 & 0,463 \\
\hline & 0,668 & 0,643 & 0,463 & 1,000 \\
\hline
\end{tabular}

TAB. 4 - Matrice DE VARIANCE-COVARIANCE ET DE CORRÉLATIONS DES EFFETS INDIVIDUELS

On remarque que tous les éléments de $\hat{\Omega}$, y compris les éléments extradiagonaux sont statistiquement différents de zéro. Cela signifie que les termes d'hétérogénéité individuels $\left(\alpha_{i 1}, \alpha_{i 2}, \alpha_{i 3}, \alpha_{i 4}\right)$ ne sont pas indépendants entre eux et que la propriété $I I A$ n'est pas vérifiée. D'ailleurs cette hypothèse d'indépendance est fortement rejetée par le test du rapport de vraisemblance effectué à partir des spécifications 2 et 3 de l'Annexe ${ }^{16}$. Comme l'on pouvait s'y

\footnotetext{
${ }^{16}$ La statistique du test de rapport de vraisemblance correspondant à l'hypothèse de nullité des corrélations
} 
attendre, les préférences individuelles pour les quatre états du marché du travail sont fortement et positivement corrélées. Les individus qui décident de participer à l'activité rémunérée auraient ainsi des caractéristiques inobservables ou des goûts communs qui se distinguent de ceux des inactifs. Par exemple, les individus ayant une préférence pour un contrat à durée déterminée auront aussi un goût prononcé pour les emplois réguliers. La corrélation la plus faible est repérée entre les aléas relatifs aux contrats à durée indéterminée et aux mesures d'insertion publiques, suggérant que les individus accédant à ces deux formes d'emplois ont moins de caractéristiques inobservables communes que ce soit en termes de préférences ou en termes de contraintes institutionnelles (nature des offres d'emploi, stéréotypes des employeurs, etc). Or la comparaison de la deuxième et troisième colonne du tableau de l'annexe montre que si l'on ne tient pas compte de ces corrélations, les coefficients associés aux états passés sont biaisés à la hausse et que la dépendance d'état est de nouveau sur-estimée. C'est pourquoi, contrairement aux précédents travaux de la littérature, l'évaluation des mesures publiques d'insertion sera ici basée sur un modèle à effets aléatoires corrélés.

\subsection{Les déterminants individuels, familiaux et conjoncturels}

Les variables explicatives ont dans leur ensemble l'effet attendu sur les probabilités d'occupation des différents états. Pour les caractéristiques individuelles, on peut tout d'abord noter que la participation au marché du travail, sous toutes ses formes (chômage, emploi précaire, emploi régulier, mesure publique d'insertion), est croissante avec l'âge et a surtout un fort impact sur le taux d'accès à un contrat à durée indéterminée. Les trajectoires d'insertion professionnelle des jeunes semblent avoir de plus en plus de chances au fil des années d'aboutir à l'obtention d'un emploi régulier. Par rapport aux individus sans diplômes - groupe de référence dans la régression -, les diplômés occuperaient davantage un emploi, mais il n'y aurait pas de réelles différences de transition entre les niveaux d'études. Le diplôme agit sans doute davantage sur la nature de l'emploi occupé (salaires, conditions de travail, etc) que sur le taux d'accès à proprement dit. Cependant, quel que soit le diplôme ou l'absence de diplôme, la probabilité d'être au chômage est identique par rapport à une référence qui reste la transition vers l'inactivité.

En ce qui concerne les différences hommes/femmes, les signes négatifs de la variable sexe indiquent que les femmes sont plus inactives que les hommes, phénomène déjà observé au tableau 3. Mais il apparaît surtout qu'elles ont une probabilité beaucoup plus faible d'occuper un emploi notamment régulier, toutes choses étant égales par ailleurs. Comme l'on pouvait s'y attendre, l'impact du nombre d'enfants sur les trajectoires d'insertion diffère entre sexes. La présence d'enfant augmenterait la probabilité de participer au marché du travail pour les hommes alors qu'elle jouerait négativement sur l'entrée dans la vie active (quel que soit le mode d'entrée) des jeunes femmes. La naissance d'un enfant conduirait certaines femmes à renoncer à leur activité rémunérée et entraînerait une substitution importante entre les contrats à durée indéterminée et l'inactivité. Ces résultats sont conformes à ceux de Blöss, Frickey \& Novi (1994) : la précocité de la maternité et la mise en couple très jeune accroît le risque d'avoir un itinéraire marqué par l'inactivité. Certaines femmes ont sans doute choisi simultanément à la fois leur trajectoire professionnelle non prioritaire et leur trajectoire familiale

entre les différents aléas est égale à 158,22. Le nombre de degrés de liberté étant au plus égal à 6 , on rejette cette hypothèse à un seuil largement inférieur à $1 \%$. 
privilégiée, peut-être même avant leur insertion à propement parlé ${ }^{17}$. D'ailleurs, il ressort que le fait de vivre en couple diminue la participation au marché du travail et notamment sous des formes précaires (mesures publiques d'insertion, emplois temporaires, chômage). Cet effet négatif est sans doute imputable aux parcours féminins ${ }^{18}$.

Les variables d'antécédents familiaux auraient peu d'impact sur le parcours suivi. Le niveau de diplôme des deux parents n'est jamais significatif ${ }^{19}$. On peut penser que le niveau d'éducation des parents influence la durée des études des enfants via une reproduction intergénérationnelle du capital scolaire [Magnac (1997)] mais qu'il n'a pas d'effet significatif direct sur les états occupés sur le marché du travail à diplôme constant. Le divorce des parents n'aurait pas non plus d'incidence sur le parcours professionnel. En revanche, les jeunes issus d'une famille nombreuse ont une probabilité plus faible d'occuper un emploi (CDD ou CDI). Cela plaiderait donc en faveur d'un certaine reproduction sociale des comportements vis à vis du marché du travail, puisque dans les familles nombreuses, l'un des parents -et en particulier la mère- a plus de chances d'être inactif.

Enfin, l'insertion des jeunes sur le marché du travail semble avoir une forte composante conjoncturelle. Leur probabilité d'être au chômage fluctue d'une année sur l'autre au rythme des essoufflements et rebonds de la croissance économique. Les variables dichotomiques temporelles semblent capter la dynamique conjoncturelle du chômage. Pour cet état, les coefficients des années 1985 à 1996, statistiquement significatifs et négatifs, s'expliquent par le fait que le chômage a atteint son point culminant (12,3\% en moyenne et $28,1 \%$ pour les moins de 25 ans) en 1997, année de référence. Les retournements de conjoncture en 1990 et 1995 sont légèrement perceptibles dans les paramètres : pour ces deux années, les coefficients associés au chômage diminuent mais pas de façon significative.

\subsection{Evaluation de l'efficacité des mesures publiques d'insertion}

L'introduction de l'état occupé l'année précédente comme variable explicative des probabilités d'occupation des statuts permet de savoir si le passage par certains états, et en particulier par un programme gouvernemental, confère aux individus un avantage pour réussir leur insertion professionnelle. Selon que la dépendance d'état se révèle forte ou non, les politiques efficaces d'aide à l'insertion ne seront pas tout à fait les mêmes. En effet, quand l'hétérogénéité individuelle domine, ce sont les caractéristiques individuelles prédéterminées (faible diplôme, situation matrimoniale, nombre d'enfants comme on l'a vu précédemment) qui expliquent principalement la difficulté à s'insérer. Ainsi, les pouvoirs publics peuvent construire une politique d'insertion ciblant ces groupes en difficulté. En revanche, si la dépendance d'état domine, c'est le passé proche de l'individu sur le marché du travail qui fera que l'individu éprouvera des difficultés à s'insérer. La solution de politique efficace est alors d'offrir des passerelles entre les différents états favorables.

\footnotetext{
${ }^{17}$ Toutefois, nos estimations ne tiennent pas compte de la possible simultanéité des choix de carrière et des décisions d'avoir des enfants. Les naissances sont ici traitées comme des événements exogènes.

${ }^{18}$ Cette hypothèse est confirmée si on effectue des régressions séparées pour les hommes et les femmes : le fait de vivre en couple diminue la participation des femmes alors que l'effet inverse est trouvé pour les hommes. Ces phénomènes sont sans doute à relier aux processus de négociation au sein des ménages lors de la prise de décision d'offre de travail.

${ }^{19}$ C'est pourquoi, les résultats ne sont pas reportés ici par souci de parcimonie.
} 
La significativité et l'ampleur de la dépendance d'état peut être analysée en testant si les coefficients d'une ligne ou d'une colonne donnée du tableau 5 sont égaux. Ce tableau reporte les coefficients de transition. Pour les femmes, ils sont construits en additionnant les effets de la variable sexe, de la variable d'état en $(t-1)$ et de leur variable croisée sexe état en $(t-1)$. Si les coefficients d'une ligne sont égaux, l'état d'origine correspondant n'influence pas les taux d'accès aux différents états. Si les coefficients d'une colonne sont égaux, la destination correspondante est aussi facilement accessible à partir de tous les états. Le choix de l'inactivité comme référence n'a aucune influence sur les comparaisons entre coefficients d'une même ligne ou d'une même colonne. Par ailleurs, l'intérêt d'une spécification croisant les variables d'état passé et de sexe est de pouvoir tester facilement et de façon formelle les différences de dépendance d'état entre hommes et femmes. En effet, la référence pour les hommes et les femmes est la même (impact moyen de l'inactivité sur la transition vers l'inactivité) et le fait d'effectuer des comparaisons par ligne ou colonne revient à créer artificiellement des groupes de contrôle à comparer au groupe des traités, c'est-à-dire ceux qui ont bénéficié d'une mesure publique d'insertion.

\begin{tabular}{|l|cccc|}
\hline \multirow{2}{*}{$\mathbf{t}$} & \multicolumn{4}{|c|}{ HOMMES } \\
(t-1) & Chômage & CDD & CDI & Mesures pub. \\
\hline Etudes & 1,497 & 1,385 & 1,154 & 2,408 \\
& $(0,336)$ & $(0,329)$ & $(0,323)$ & $(0,369)$ \\
Chômage & 0,059 & $-0,456$ & $-0,973$ & 0,138 \\
& $(0,274)$ & $(0,272)$ & $(0,274)$ & $(0,328)$ \\
CDD & $-1,312$ & 0,052 & $-1,076$ & $-0,846$ \\
& $(0,275)$ & $(0,266)$ & $(0,260)$ & $(0,351)$ \\
CDI & $-1,371$ & $-1,969$ & 1,081 & $-1,650$ \\
& $(0,264)$ & $(0,269)$ & $(0,238)$ & $(0,385)$ \\
Mesures pub. & 0,113 & 0,042 & 0,302 & 2,064 \\
& $(0,299)$ & $(0,299)$ & $(0,281)$ & $(0,338)$ \\
\hline \hline \multirow{3}{*}{$\mathbf{t}$} & \multicolumn{4}{|c}{ FEMMES } \\
(t-1) & Chômage & CDD & CDI & Mesures pub. \\
\hline Etudes & 0,308 & 0,012 & $-0,408$ & 1,126 \\
& $(0,239)$ & $(0,238)$ & $(0,232)$ & $(0,281)$ \\
Chômage & 1,605 & 0,539 & $-0,116$ & 1,777 \\
& $(0,294)$ & $(0,295)$ & $(0,291)$ & $(0,340)$ \\
CDD & 0,191 & 0,966 & $-0,399$ & 0,087 \\
& $(0,282)$ & $(0,282)$ & $(0,273)$ & $(0,349)$ \\
CDI & $-0,085$ & $-1,211$ & 2,020 & $-0,041$ \\
& $(0,291)$ & $(0,305)$ & $(0,277)$ & $(0,371)$ \\
Mesures pub. & 0,719 & 0,511 & $-0,063$ & 2,399 \\
& $(0,298)$ & $(0,299)$ & $(0,298)$ & $(0,332)$ \\
\hline
\end{tabular}

les écarts-types sont reportés entre parenthèses.

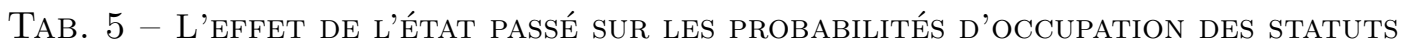

Des tests de Wald effectués sur chaque ligne et chaque colonne rejettent l'hypothèse d'absence de dépendance d'état, quelle que soit la situation d'origine ou de destination. Par exemple, occuper un emploi régulier diminue la probabilité d'accéder à un autre état la période suivante. De même, les contrats à durée déterminée et les mesures publiques d'insertion apparaissent comme des états où les transitions vers les autres situations sont toujours moins probables que le maintien dans ces états ${ }^{20}$. Ces deux phénomènes confirment une segmen-

\footnotetext{
${ }^{20}$ Pour les hommes en provenance d'un contrat à durée déterminée, la probabilité de rester dans cet état
} 
tation du marché du travail entre emplois précaires et emplois réguliers et seraient donc la conséquence du dualisme du marché. Les taux de transition vers l'emploi régulier, le chômage ou une mesure publique d'insertion, en provenance des contrats à durée déterminée, sont d'ailleurs statistiquement identiques.

Pour les chômeurs, l'accès à un programme gouvernemental d'insertion semble aussi probable que de rester au chômage. Mais en revanche, ils ont de grandes difficultés à accéder à un emploi temporaire et de plus grandes difficultés encore à transiter vers un emploi régulier. Les jeunes semblent donc trouver dans les mesures publiques un refuge contre le chômage. C'est pourquoi, ces mesures sont une issue aux études très fréquente. Cela confirme les obstacles et la précarité rencontrés par de nombreux jeunes au début de leur vie active. En fait, la structure d'entrée dans les programmes gouvernementaux est très semblable (pas de différence significative) à celle du chômage bien qu'elle soit plus accessible à partir des études ou d'un précédent programme.

\subsubsection{Influence des mesures publiques sur l'accès à un emploi stable}

Pour les hommes, les mesures publiques semblent avoir un effet bénéfique sur l'insertion en contrat à durée indéterminée par rapport au chômage et aux emplois temporaires. Si on teste la similarité entre l'insertion en provenance des mesures publiques et du chômage ou entre mesures publiques et CDD, les différences sont statistiquement significatives à un seuil de $1 \%^{21}$. Les programmes gouvernementaux seraient ainsi de meilleurs tremplins vers l'emploi stable que le chômage ou les contrats à durée déterminée pour les hommes. Cependant en cas d'échec de l'insertion, ils ont plus de chances de conduire au chômage que les emplois temporaires ${ }^{22}$. En conséquence, l'avantage que confère la participation à une mesure publique par rapport à un contrat à durée déterminée peut être à double tranchant. Mais il paraît incontestable que le passage par une telle mesure permet aux jeunes hommes au chômage de mieux s'insérer : ces stages facilitent leur accès à un emploi régulier sans diminuer leurs chances d'accéder à un emploi temporaire ni augmenter leurs chances de rester au chômage ${ }^{23}$.

En revanche, pour les femmes, le passage par un programme gouvernemental ne facilite pas leur insertion en contrat à durée indéterminée. Leurs chances d'accéder à un emploi régulier sont identiques, qu'elles soient précédemment en situation de chômage, en emplois précaires, en mesures publiques et même en inactivité ${ }^{24}$. Ainsi, la participation au marché du travail sous une forme précaire (chômage, mesures publiques d'insertion et emplois temporaires) ne

n'est pas statistiquement différente de celle de transiter vers l'inactivité.

${ }^{21}$ Les statistiques des tests de Wald sont respectivement égales à 18,64 et 27,67 pour une valeur critique de $\chi_{.01}^{2}(1)=6,63$.

${ }^{22}$ L'hypothèse $\delta_{\text {mesure pub.-chômage }}=\delta_{\text {cdd-chômage }}$ est rejetée à un seuil de $1 \%$.

${ }^{23}$ Les facilités d'accès en provenance d'une mesure publique ou d'un CDD ne sont pas statistiquement différentes. De même, les coefficients de transition d'une mesure publique vers le chômage et du maintien en chômage sont identiques.

${ }^{24}$ Les statistiques correspondant aux tests de la similarité entre l'insertion en provenance des mesures publiques et du chômage ou entre mesures publiques et CDD ou mesures publiques et études sont respectivement égales à $0,03,1,26$ et 1,77 pour une valeur critique de $\chi_{.01}^{2}(1)=6,63$. En outre, les coefficients de la colonne CDI ne sont pas significatifs par rapport à l'état de référence, l'inactivité, hormis celui de la transition CDI vers CDI. 
serait pas un atout en comparaison à l'inactivité pour réussir son insertion. Summers \& Clark (1990) et Jones \& Riddell (1999) ont déjà mis en évidence des résultats très proches. Summers \& Clark (1990) concluaient que pour les jeunes, les taux d'accès à l'emploi en provenance du chômage et de l'inactivité ne pouvaient pas être distingués et Jones \& Riddell (1999) ont montré que certaines catégories d'inactifs avaient les mêmes chances de trouver un emploi que les chômeurs. Par ailleurs, il faut garder en tête que dans notre modélisation, les effets des états passés sont nets de l'hétérogénéité individuelle inobservée. Or dans les faits, ce qui différencie principalement une chômeuse d'une femme inactive est à relier à leurs préférences respectives pour le travail, préférences qui sont captées par les termes d'hétérogénéité.

La faible fréquence d'accès à l'emploi stable à partir des emplois précaires et leur nonsignificativité par rapport à l'inactivité est aussi le reflet des effets de découragement et de sorties du marché du travail des femmes qui n'ont pas pu accéder à un contrat à durée indéterminée. En revanche, quand elles arrivent à décrocher ce type de contrat, elles y restent plus longtemps que les hommes.

Comme Magnac (1997, 2000 b), on réfute donc que les mesures publiques d'insertion sont des tremplins vers l'emploi stable quand on tient compte de l'hétérogénéité inobservée, mais uniquement dans le cas des femmes. Parmi les hommes qui ont des difficultés d'insertion, ceux qui suivent une mesure publique ont plus de chances d'obtenir un emploi stable que les chômeurs alors qu'une chômeuse a autant de chances d'y arriver qu'une femme bénéficiant d'un tel programme. Mais cela ne signifie pas que les dispositifs publics favorisent l'insertion des jeunes hommes au détriment de celle des jeunes femmes. En effet, en comparant les deux lignes en mesures publiques en ( $t$-1) du tableau 5, il apparaît que les probabilités conditionnelles de rester en programmes gouvernementaux et de transiter vers un autre état sont identiques pour les deux sexes ${ }^{25}$. En particulier, les taux d'accès des hommes et des femmes à un emploi régulier en provenance d'une mesure publique sont statistiquement égaux. Ainsi, un homme suivant une telle mesure a autant de chances qu'une femme suivant aussi une mesure publique ou qu'une chômeuse d'obtenir un emploi stable, mais tous ont un avantage vis-à-vis des hommes au chômage.

\subsubsection{Le passage par le chômage ou un CDD plus handicapant chez les hommes pour l'obtention d'un CDI}

En effet, le passage par une situation de chômage ou par un emploi temporaire semble être plus handicapant pour les hommes que pour les femmes en termes d'insertion. Par exemple, une chômeuse a une probabilité plus élevée qu'un homme au chômage d'obtenir un contrat à durée déterminée ou d'accéder à un emploi régulier ${ }^{26}$. De même, parmi les jeunes en emplois temporaires, les femmes ont une plus grande facilité à transiter vers un CDI. Les épisodes de précarité (chômage, CDD) des hommes agiraient donc comme de mauvais signaux pour leurs futurs employeurs potentiels. Au contraire, la précarité des femmes ne semble pas être perçue comme un signal de faible performance dans l'emploi. Ce résultat pourrait être le reflet d'une certaine forme de discrimination statistique : en raison de leurs préjugés ou de

\footnotetext{
${ }^{25}$ Les statistiques des tests de Wald des coefficients pris deux à deux sont respectivement de 3,16, 2,25, 1,41, 1,13 .

${ }^{26}$ Les statistiques correspondantes aux tests d'égalité de ces deux coefficients entre sexes sont respectivement de 11,79 et 8,62 .
} 
leurs observations antérieures du marché du travail, les employeurs considèrent sans doute la précarité comme une situation plus habituelle (ou plus « normale ») pour les femmes et donc ils tiendraient moins compte de cette information lors de leurs recrutements puisque ces épisodes de précarité seraient moins révélateurs de leur niveau de compétences que dans le cas des hommes. Bonnal et al. (1997) ont déjà observé ces phénomènes de signaux mais pour des groupes différents : ils trouvaient que pour les plus éduqués, le passage par des programmes de formation diminuait l'intensité de transition d'un épisode de chômage vers l'emploi régulier car cette transition peu fréquente pour ce groupe était interprétée comme un signal de mauvaise performance.

\subsubsection{L'obtention d'un emploi protège moins les femmes contre le chômage}

Néanmoins, même si la dépendance d'état pour accéder à un contrat à durée indéterminée est plus bénéfique aux femmes, il n'en reste pas moins qu'elles ont plus de mal à s'insérer. A la fin de leurs études, elles sont moins nombreuses à débuter directement leurs carrières par un emploi régulier. Par ailleurs, si elles arrivent à obtenir un emploi temporaire, une mesure publique d'insertion ou un contrat à durée indéterminée, elles ont une probabilité plus élevée de se retrouver au chômage l'année suivante comparativement aux hommes dans les mêmes situations. Or, une fois au chômage, les femmes y restent significativement plus longtemps que les hommes. Par ailleurs, comme ce fut évoqué précédemment, il est beaucoup plus difficile pour les chômeurs - et ce pour les deux sexes - d'accéder à un emploi régulier qu'aux autres formes d'emploi.

En résumé, contrairement aux hommes, les jeunes femmes utilisent sans doute les mesures publiques d'insertion comme un refuge contre le chômage plutôt que pour leurs qualités insérantes. Il est vrai que pour les jeunes femmes, l'insertion sur le marché du travail est singulièrement difficile. Mais cela peut aussi être lié au type de mesures publiques qu'on leur offre, compte tenu de leur formation initiale : il s'agit plus fréquemment de « contrats d'emploi solidarité » dans la fonction publique ou d'emplois subventionnés dans le secteur non marchand que de stages de qualification en entreprise privée. Cependant, du point de vue des employeurs, la précarité féminine aurait une moindre connotation négative que chez les hommes. Si la précarité féminine est mieux acceptée lors des recrutements, c'est probablement que les jeunes femmes ont plus de mal à en sortir et qu'elle est presque devenue la règle lors de leur entrée dans la vie active. Mais en contrepartie, il est aussi plus facile pour les femmes

d'y être de nouveau confrontées après un épisode d'emploi régulier. Les parcours d'insertion des femmes seraient donc plus instables. Ils le sont d'autant plus que lors de ces années-là, un changement de situation matrimoniale ou la naissance d'un enfant peut venir affecter leurs comportements professionnels : en particulier ces événements familiaux freine l'accès à un CDI chez les femmes.

\section{Conclusion}

Cet article propose une étude de l'insertion professionnelle des jeunes qui détaille les trajectoires différenciées entre hommes et femmes. Son objectif principal est d'évaluer, pour ces deux groupes, l'efficacité des mesures publiques (contrats aidés, programme de formation professionnelle), c'est-à-dire de voir si ces dernières favorisent l'accès à des emplois stables. Pour 
ce faire, on a utilisé une méthode quantitative adaptée aux données d'enquête longitudinales portant sur des trajectoires entre états discrets sur le marché du travail. En particulier, on a estimé, par maximum de vraisemblance simulé, un logit polytomique dynamique qui tient compte des effets corrélés d'hétérogénéité individuelle, ce qui n'est pas encore très répandu dans la littérature d'économie du travail. L'avantage de cette modélisation est qu'elle permet d'identifier les caractéristiques individuelles défavorables à l'insertion professionnelle et de savoir si les effets de la dépendance d'état jouent un rôle important ou en d'autres termes si le passé proche d'un individu peut lui faire éprouver des difficultés supplémentaires. La distinction entre hétérogénéité individuelle et dépendance d'état est un cadre d'investigation utile pour préconiser des politiques économiques efficaces. Si l'hétérogénéité prédomine, ce sont des caractéristiques individuelles particulières (diplôme, sexe, etc) qui expliquent principalement la difficulté de s'insérer. La stratégie efficace pour les pouvoirs publics est alors de cibler leurs mesures sur les groupes en difficulté. En revanche, si c'est la dépendance d'état qui domine, la politique appropriée serait de chercher à modifier le fonctionnement du marché du travail en créant des passerelles entre les différents états, via par exemple des aides à la recherche d'emploi, des stages en entreprises.

Les estimations effectuées à partir des données françaises de l'Enquête Jeunes et Carrières 1997 montrent que les facteurs handicapant l'insertion des jeunes diffèrent entre sexes. Chez les hommes, il semble que c'est la dépendance d'état qui est prédominante pour obtenir un emploi régulier. Notamment le passage par un emploi temporaire ou un épisode de chômage risque d'être interprété comme un signal de faible performance et ainsi risque de limiter les chances de l'individu d'accéder à un contrat à durée indéterminée ultérieurement. Dans ce contexte de forte dépendance d'état, il apparaît que les mesures publiques d'insertion ont un effet bénéfique sur l'insertion en emploi stable.

En revanche, chez les femmes, l'état occupé l'année précédente n'a pas d'influence significative sur le taux d'accès à un contrat à durée indéterminée. Ainsi, les employeurs pratiqueraient une forme de discrimination statistique lors de l'embauche : le poids accordé à l'historique professionnel et notamment aux passages par la précarité serait moins important chez les femmes, comme s'ils reconnaissaient implicitement que la précarité féminine est une situation plus habituelle et donc moins révélatrice de leur niveau de compétences que dans le cas des hommes. Chez les femmes, Ce serait davantage les caractéristiques individuelles qui expliqueraient les disparités de trajectoires professionnelles. En particulier, le manque de qualification et le fait d'avoir des enfants sont autant de facteurs qui diminuent leur probabilité d'obtenir un emploi stable et de manière plus générale de participer au marché du travail. Ainsi, pour les femmes, les programmes de formation offrant des passerelles vers l'emploi stable ne pourront sans doute être totalement efficaces que s'ils sont couplés avec des politiques favorisant la conciliation entre vie professionnelle et vie familiale (réorganisation du temps de travail, politique sur les gardes d'enfants, etc). Leur but devrait être de réduire l'instabilité des parcours féminins.

Afin d'améliorer la robustesse statistique des évaluations, on pourrait envisager d'endogénéiser les variables d'enfants. De nombreuses femmes décident vraisemblablement de leurs parcours professionnels et familiaux de façon simultanée. Du point de vue de l'estimation, cela reviendrait à redéfinir chaque état de transition comme un couplé formé de la situation professionnelle de l'individu et de son nombre d'enfants. Or dans nos données, peu 
de jeunes avaient des enfants et donc cela risquait de poser des problèmes d'identification. Une piste de recherche intéressante serait par conséquent de reproduire le même genre de modélisations économétriques des trajectoires professionnelles mais sur des populations plus âgées en tenant compte de l'endogénéité des enfants. 


\section{Annexe}

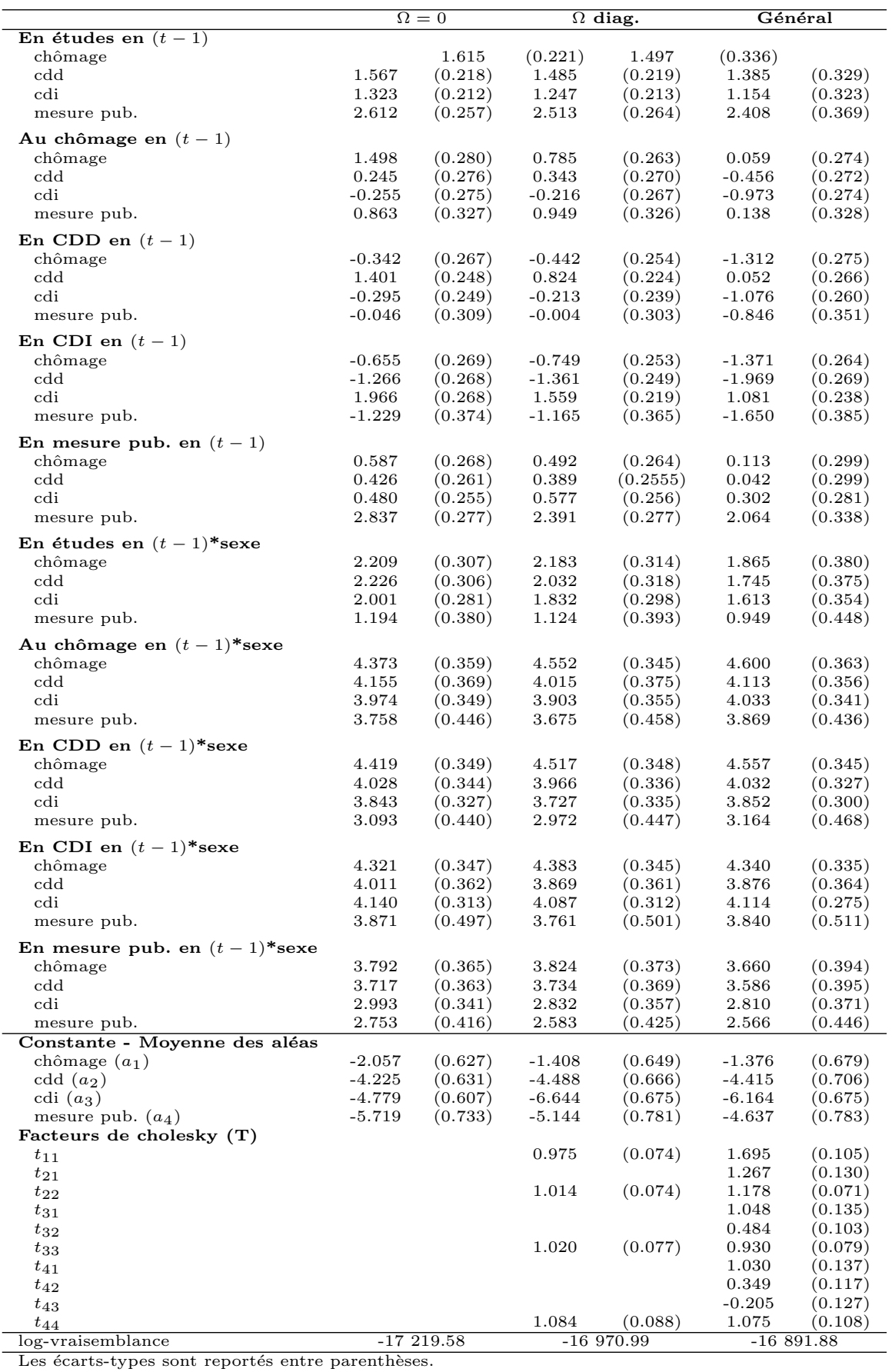

TAB. 6 - INFLUENCE DE LA DÉPENDANCE D'ÉTAT ET DE L'HÉTÉROGÉNÉITÉ SUR LES PROBABILITÉS D'OCCUPATION DES STATUTS 


\begin{tabular}{|c|c|c|c|c|c|c|}
\hline \multirow[b]{2}{*}{ Age } & \multicolumn{2}{|c|}{$\Omega=0$} & \multicolumn{2}{|c|}{$\Omega$ diag. } & \multicolumn{2}{|c|}{ Général } \\
\hline & & & & & & \\
\hline chômage & 1.478 & $(0.290)$ & 1.220 & $(0.298)$ & 1.744 & $(0.284)$ \\
\hline cdd & 2.210 & $(0.291)$ & 2.295 & $(0.303)$ & 2.812 & $(0.294)$ \\
\hline cdi & 2.513 & $(0.283)$ & 3.381 & $(0.300)$ & 3.681 & $(0.277)$ \\
\hline mesure pub. & 2.177 & $(0.333)$ & 1.726 & $(0.357)$ & 1.997 & $(0.320)$ \\
\hline \multicolumn{7}{|c|}{ Sexe $($ femme $=1)$} \\
\hline chômage & -3.162 & $(0.279)$ & -3.130 & $(0.280)$ & -3.054 & $(0.267)$ \\
\hline cdd & -3.288 & $(0.282)$ & -3.158 & $(0.284)$ & -3.117 & $(0.265)$ \\
\hline cdi & -3.247 & $(0.254)$ & -3.193 & $(0.260)$ & -3.175 & $(0.229)$ \\
\hline mesure pub. & -2.309 & $(0.353)$ & -2.201 & $(0.362)$ & -2.231 & $(0.342)$ \\
\hline \multicolumn{7}{|l|}{ En couple } \\
\hline chômage & -0.451 & $(0.095)$ & -0.456 & $(0.104)$ & -0.570 & $(0.126)$ \\
\hline cdd & -0.380 & $(0.095)$ & -0.455 & $(0.102)$ & -0.556 & $(0.124)$ \\
\hline cdi & -0.048 & $(0.089)$ & -0.009 & $(0.096)$ & -0.131 & $(0.118)$ \\
\hline mesure pub. & -0.475 & $(0.114)$ & -0.541 & $(0.126)$ & -0.662 & $(0.153)$ \\
\hline \multicolumn{7}{|c|}{ Nbre d'enfants } \\
\hline chômage & 0.382 & $(0.402)$ & 0.463 & $(0.384)$ & 0.611 & $(0.203)$ \\
\hline cdd & 0.390 & $(0.394)$ & 0.399 & $(0.378)$ & 0.551 & $(0.198)$ \\
\hline cdi & 0.325 & $(0.379)$ & 0.386 & $(0.364)$ & 0.511 & $(0.182)$ \\
\hline mesure pub. & 0.467 & $(0.413)$ & 0.533 & $(0.404)$ & 0.691 & $(0.288)$ \\
\hline \multicolumn{7}{|c|}{ Nbre d'enfants*sexe } \\
\hline chômage & -1.112 & $(0.407)$ & -1.257 & $(0.391)$ & -1.730 & $(0.217)$ \\
\hline cdd & -1.385 & $(0.403)$ & -1.569 & $(0.390)$ & -2.036 & $(0.216)$ \\
\hline cdi & -1.519 & $(0.387)$ & -1.798 & $(0.374)$ & -2.188 & $(0.196)$ \\
\hline mesure pub. & -1.603 & $(0.428)$ & -1.670 & $(0.426)$ & -2.093 & $(0.311)$ \\
\hline \multicolumn{7}{|c|}{ Enseign.profesionnel court } \\
\hline chômage & 0.265 & $(0.106)$ & 0.211 & $(0.116)$ & 0.358 & $(0.144)$ \\
\hline cdd & 0.603 & $(0.106)$ & 0.694 & $(0.117)$ & 0.826 & $(0.144)$ \\
\hline cdi & 0.851 & $(0.100)$ & 1.006 & $(0.113)$ & 1.100 & $(0.135)$ \\
\hline mesure pub. & 0.293 & $(0.125)$ & 0.368 & $(0.136)$ & 0.454 & $(0.156)$ \\
\hline \multicolumn{7}{|c|}{ Enseign.profesionnel long } \\
\hline chômage & 0.051 & $(0.176)$ & -0.023 & $(0.190)$ & 0.069 & $(0.218)$ \\
\hline cdd & 0.488 & $(0.173)$ & 0.576 & $(0.192)$ & 0.647 & $(0.215)$ \\
\hline cdi & 0.776 & $(0.165)$ & 0.860 & $(0.182)$ & 0.919 & $(0.202)$ \\
\hline mesure pub. & 0.290 & $(0.203)$ & 0.374 & $(0.223)$ & 0.428 & $(0.234)$ \\
\hline \multicolumn{7}{|l|}{ Bac général } \\
\hline chômage & 0.003 & $(0.165)$ & -0.081 & $(0.176)$ & 0.029 & $(0.206)$ \\
\hline cdd & 0.578 & $(0.164)$ & 0.674 & $(0.178)$ & 0.756 & $(0.199)$ \\
\hline cdi & 0.993 & $(0.157)$ & 1.091 & $(0.171)$ & 1.167 & $(0.189)$ \\
\hline mesure pub. & 0.170 & $(0.201)$ & 0.227 & $(0.217)$ & 0.289 & $(0.220)$ \\
\hline \multicolumn{7}{|c|}{ Au moins $\mathrm{Bac}+2$} \\
\hline chômage & 0.002 & $(0.271)$ & 0.018 & $(0.289)$ & 0.004 & $(0.336)$ \\
\hline cdd & 0.250 & $(0.277)$ & 0.145 & $(0.294)$ & 0.168 & $(0.336)$ \\
\hline cdi & 1.005 & $(0.258)$ & 1.047 & $(0.276)$ & 1.028 & $(0.315)$ \\
\hline mesure pub. & 0.231 & $(0.323)$ & 0.330 & $(0.342)$ & 0.328 & $(0.350)$ \\
\hline \multicolumn{7}{|c|}{ Nbre de frères et sœurs } \\
\hline $\begin{array}{l}\text { chômage } \\
\text { cdd }\end{array}$ & $\begin{array}{c}0.024 \\
-0.038\end{array}$ & $\begin{array}{l}(0.019) \\
(0.021)\end{array}$ & $\begin{array}{c}0.034 \\
-0.053\end{array}$ & $\begin{array}{l}(0.021) \\
(0.023)\end{array}$ & $\begin{array}{c}0.012 \\
-0.071\end{array}$ & $\begin{array}{l}(0.025) \\
(0.027)\end{array}$ \\
\hline cdi & -0.052 & $(0.019)$ & -0.071 & $(0.022)$ & -0.084 & $(0.023)$ \\
\hline mesure pub. & -0.009 & $(0.022)$ & -0.013 & $(0.025)$ & -0.024 & $(0.029)$ \\
\hline \multicolumn{7}{|c|}{ Parents divorcés } \\
\hline chômage & 0.143 & $(0.115)$ & 0.196 & $(0.123)$ & 0.196 & $(0.145)$ \\
\hline cdd & 0.064 & $(0.116)$ & 0.073 & $(0.127)$ & 0.072 & $(0.144)$ \\
\hline cdi & -0.060 & $(0.110)$ & -0.067 & $(0.119)$ & -0.054 & $(0.136)$ \\
\hline mesure pub. & 0.108 & $(0.133)$ & 0.144 & $(0.144)$ & 0.144 & $(0.163)$ \\
\hline
\end{tabular}

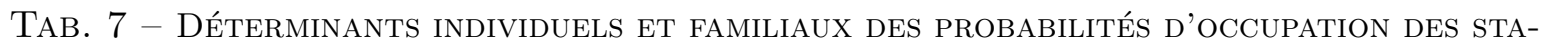
TUTS 


\begin{tabular}{|c|c|c|c|c|c|c|}
\hline & \multicolumn{2}{|c|}{$\Omega=0$} & \multicolumn{2}{|c|}{$\Omega$ diag. } & \multicolumn{2}{|c|}{ Général } \\
\hline $\begin{array}{l}\text { Année } 1985 \\
\text { chômage } \\
\text { cdd } \\
\text { cdi } \\
\text { mesure pub. }\end{array}$ & $\begin{array}{c}-2.042 \\
-0.432 \\
0.721 \\
-0.284\end{array}$ & $\begin{array}{l}(0.503) \\
(0.505) \\
(0.391) \\
(0.516)\end{array}$ & $\begin{array}{c}-2.675 \\
-0.215 \\
1.271 \\
-0.230\end{array}$ & $\begin{array}{l}(0.528) \\
(0.540) \\
(0.430) \\
(0.573)\end{array}$ & $\begin{array}{c}-2.601 \\
-0.264 \\
1.133 \\
-0.395\end{array}$ & $\begin{array}{l}(0.756) \\
(0.705) \\
(0.592) \\
(0.669)\end{array}$ \\
\hline $\begin{array}{l}\text { Année } 1986 \\
\text { chômage } \\
\text { cdd } \\
\text { cdi } \\
\text { mesure pub. }\end{array}$ & $\begin{array}{c}-1.767 \\
-0.081 \\
0.078 \\
-0.133\end{array}$ & $\begin{array}{l}(0.331) \\
(0.312) \\
(0.325) \\
(0.368)\end{array}$ & $\begin{array}{c}-2.174 \\
0.096 \\
0.461 \\
-0.243\end{array}$ & $\begin{array}{l}(0.366) \\
(0.340) \\
(0.350) \\
(0.401)\end{array}$ & $\begin{array}{c}-2.250 \\
-0.058 \\
0.281 \\
-0.427\end{array}$ & $\begin{array}{l}(0.467) \\
(0.460) \\
(0.434) \\
(0.487)\end{array}$ \\
\hline $\begin{array}{l}\text { Année } 1987 \\
\text { chômage } \\
\text { cdd } \\
\text { cdi } \\
\text { mesure pub. }\end{array}$ & $\begin{array}{l}-1.385 \\
0.252 \\
0.595 \\
0.411\end{array}$ & $\begin{array}{l}(0.269) \\
(0.265) \\
(0.256) \\
(0.293)\end{array}$ & $\begin{array}{c}-1.788 \\
0.390 \\
0.910 \\
0.463\end{array}$ & $\begin{array}{l}(0.291) \\
(0.280) \\
(0.275) \\
(0.314)\end{array}$ & $\begin{array}{l}-1.826 \\
0.285 \\
0.746 \\
0.289\end{array}$ & $\begin{array}{l}(0.376) \\
(0.362) \\
(0.349) \\
(0.382)\end{array}$ \\
\hline $\begin{array}{l}\text { Année } 1988 \\
\text { chômage } \\
\text { cdd } \\
\text { cdi } \\
\text { mesure pub. }\end{array}$ & $\begin{array}{l}-1.380 \\
0.264 \\
0.373 \\
0.357\end{array}$ & $\begin{array}{l}(0.243) \\
(0.235) \\
(0.232) \\
(0.267)\end{array}$ & $\begin{array}{l}-1.725 \\
0.370 \\
0.598 \\
0.404\end{array}$ & $\begin{array}{l}(0.257) \\
(0.252) \\
(0.249) \\
(0.288)\end{array}$ & $\begin{array}{l}-1.822 \\
0.217 \\
0.401 \\
0.203\end{array}$ & $\begin{array}{l}(0.305) \\
(0.283) \\
(0.270) \\
(0.317)\end{array}$ \\
\hline $\begin{array}{l}\text { Année } 1989 \\
\text { chômage } \\
\text { cdd } \\
\text { cdi } \\
\text { mesure pub. }\end{array}$ & $\begin{array}{c}-1.076 \\
0.330 \\
0.639 \\
0.021\end{array}$ & $\begin{array}{l}(0.213) \\
(0.205) \\
(0.206) \\
(0.245)\end{array}$ & $\begin{array}{r}-1.367 \\
0.420 \\
0.833 \\
-0.038\end{array}$ & $\begin{array}{l}(0.228) \\
(0.220) \\
(0.221) \\
(0.263)\end{array}$ & $\begin{array}{c}-1.475 \\
0.270 \\
0.662 \\
-0.218\end{array}$ & $\begin{array}{l}(0.267) \\
(0.263) \\
(0.246) \\
(0.309)\end{array}$ \\
\hline $\begin{array}{l}\text { Année } 1990 \\
\text { chômage } \\
\text { cdd } \\
\text { cdi) } \\
\text { mesure pub. }\end{array}$ & $\begin{array}{c}-1.233 \\
0.221 \\
0.544 \\
-0.123\end{array}$ & $\begin{array}{l}(0.196) \\
(0.191) \\
(0.184) \\
(0.233)\end{array}$ & $\begin{array}{r}-1.499 \\
0.319 \\
0.783 \\
-0.219\end{array}$ & $\begin{array}{l}(0.210) \\
(0.205) \\
(0.199) \\
(0.252)\end{array}$ & $\begin{array}{c}-1.568 \\
0.210 \\
0.643 \\
-0.376\end{array}$ & $\begin{array}{l}(0.242) \\
(0.234) \\
(0.224) \\
(0.280)\end{array}$ \\
\hline $\begin{array}{l}\text { Année } 1991 \\
\text { chômage } \\
\text { cdd } \\
\text { cdi } \\
\text { mesure pub. }\end{array}$ & $\begin{array}{c}-0.891 \\
0.174 \\
0.544 \\
0.044\end{array}$ & $\begin{array}{l}(0.178) \\
(0.178) \\
(0.171) \\
(0.216)\end{array}$ & $\begin{array}{c}-1.158 \\
0.259 \\
0.774 \\
-0.071\end{array}$ & $\begin{array}{l}(0.190) \\
(0.191) \\
(0.184) \\
(0.234)\end{array}$ & $\begin{array}{c}-1.228 \\
0.152 \\
0.641 \\
-0.227\end{array}$ & $\begin{array}{l}(0.224) \\
(0.221) \\
(0.210) \\
(0.265)\end{array}$ \\
\hline $\begin{array}{l}\text { Année } 1992 \\
\text { chômage } \\
\text { cdd } \\
\text { cdi } \\
\text { mesure pub. }\end{array}$ & $\begin{array}{l}-0.930 \\
0.226 \\
0.482 \\
0.323\end{array}$ & $\begin{array}{l}(0.169) \\
(0.167) \\
(0.159) \\
(0.197)\end{array}$ & $\begin{array}{c}-1.156 \\
0.310 \\
0.684 \\
0.297\end{array}$ & $\begin{array}{l}(0.180) \\
(0.179) \\
(0.171) \\
(0.213)\end{array}$ & $\begin{array}{l}-1.182 \\
0.245 \\
0.591 \\
0.194\end{array}$ & $\begin{array}{l}(0.207) \\
(0.205) \\
(0.197) \\
(0.247)\end{array}$ \\
\hline $\begin{array}{l}\text { Année } 1993 \\
\text { chômage } \\
\text { cdd } \\
\text { cdi } \\
\text { mesure pub. }\end{array}$ & $\begin{array}{c}-0.548 \\
0.431 \\
0.573 \\
0.676\end{array}$ & $\begin{array}{l}(0.155) \\
(0.156) \\
(0.148) \\
(0.186)\end{array}$ & $\begin{array}{l}-0.742 \\
0.507 \\
0.726 \\
0.676\end{array}$ & $\begin{array}{l}(0.163) \\
(0.166) \\
(0.156) \\
(0.203)\end{array}$ & $\begin{array}{c}-0.752 \\
0.464 \\
0.658 \\
0.600\end{array}$ & $\begin{array}{l}(0.208) \\
(0.199) \\
(0.199) \\
(0.232)\end{array}$ \\
\hline $\begin{array}{l}\text { Année } 1994 \\
\text { chômage } \\
\text { cdd } \\
\text { cdi } \\
\text { mesure pub. }\end{array}$ & $\begin{array}{l}-0.467 \\
0.323 \\
0.285 \\
0.193\end{array}$ & $\begin{array}{l}(0.144) \\
(0.145) \\
(0.139) \\
(0.180)\end{array}$ & $\begin{array}{c}-0.613 \\
0.403 \\
0.388 \\
0.156\end{array}$ & $\begin{array}{l}(0.152) \\
(0.154) \\
(0.147) \\
(0.198)\end{array}$ & $\begin{array}{l}-0.627 \\
0.358 \\
0.337 \\
0.089\end{array}$ & $\begin{array}{l}(0.190) \\
(0.192) \\
(0.184) \\
(0.225)\end{array}$ \\
\hline $\begin{array}{l}\text { Année } 1995 \\
\text { chômage } \\
\text { cdd } \\
\text { cdi } \\
\text { mesure pub. }\end{array}$ & $\begin{array}{l}-0.655 \\
0.114 \\
0.160 \\
0.077\end{array}$ & $\begin{array}{l}(0.137) \\
(0.138) \\
(0.130) \\
(0.170)\end{array}$ & $\begin{array}{c}-0.764 \\
0.168 \\
0.230 \\
0.036\end{array}$ & $\begin{array}{l}(0.143) \\
(0.147) \\
(0.136) \\
(0.187)\end{array}$ & $\begin{array}{c}-0.785 \\
0.125 \\
0.185 \\
-0.023\end{array}$ & $\begin{array}{l}(0.178) \\
(0.181) \\
(0.176) \\
(0.213)\end{array}$ \\
\hline $\begin{array}{l}\text { Année } 1996 \\
\text { chômage } \\
\text { cdd } \\
\text { cdi } \\
\text { mesure pub. }\end{array}$ & $\begin{array}{l}-0.546 \\
0.015 \\
0.149 \\
0.067\end{array}$ & $\begin{array}{l}(0.123) \\
(0.123) \\
(0.116) \\
(0.152)\end{array}$ & $\begin{array}{c}-0.623 \\
0.047 \\
0.209 \\
0.050\end{array}$ & $\begin{array}{l}(0.127) \\
(0.129) \\
(0.121) \\
(0.166)\end{array}$ & $\begin{array}{c}-0.626 \\
0.030 \\
0.187 \\
0.017\end{array}$ & $\begin{array}{l}(0.188) \\
(0.195) \\
(0.186) \\
(0.226)\end{array}$ \\
\hline
\end{tabular}

TAB. 8 - Déterminants temporels des PRobabilités D'ocCupation des STATUtS 


\section{Références}

Andersen, E. (1973), Conditional Inference and Models for Measuring, Copenhaguen : Metalhygienjnisk Vorlag.

Anxo, D., Flood, L. \& Kocoglu, Y. (2002), 'Offre de travail et répartition des activités domestiques et parentales au sein du couple : une comparaison entre la France et la Suède', Économie et Statistique 352-353, 127-150.

Blöss, T., Frickey, A. \& Novi, M. (1994), 'Modes d'entrées dans la vie adulte et trajectoires sociales des femmes mariées', Population 3(mai-juin), 637-656.

Bonnal, L., Fougère, D. \& Sérandon, A. (1994), 'L'impact des dispositifs d'emploi sur le devenir des jeunes chômeurs : une évaluation économétrique sur données longitudinales', Économie et Prévision 115(4), 1-28.

Bonnal, L., Fougère, D. \& Sérandon, A. (1997), 'Evaluating the Impact of French Policies on Individual Labour Markets Histories', Review of Economic Studies 64(4), 683-713.

Brodaty, T., Crépon, B. \& Fougère, D. (2001), Using Matching Estimators to Evaluate Alternative youth Employment Program : Evidence from France, 1986-1988, in M. Lechner \& F. Pfeiffer, eds, 'Econometric Evaluations of Active Labor Market Policies in Europe', Physica-Verlag : Heidelberg, pp. 43-58.

Card, D. \& Hyslop, D. (2002), 'Estimating the Dynamic Treatment Effect of an Earnings Subsidy for Welfare-Leavers', Center for Labor Economics - University of California Berkeley Working Paper 47 (March).

Card, D. \& Sullivan, D. (1988), 'Measuring the Effect of Subsidized Training Programs on Movements In and Out of Employment', Econometrica 56(3), 497-530.

Cases, C. \& Lollivier, S. (1994), 'Estimation d'un modèle de sortie de chômage à destinations multiples', Economie et Prévision 2-3(113-114), 177-188.

Chamberlain, G. (1984), Panel Data, in Z. Grilliches \& M. Intriligator, eds, 'Handbook of Econometrics', Vol. 2, Elsevier, North-Holland, pp. 1247-1318.

DARES (1996), 40 ans de politiques de l'emploi, La documentation franccaise, Paris.

Fougère, D. (1998), 'Expérimenter pour évaluer les politiques d'aide à l'emploi : les exemples anglo-saxons et nord-européens', Revue française des Affaires sociales 54(1), 111-144.

Fougère, D., Kramarz, F. \& Magnac, T. (2000), 'Youth Employment Policies in France', European Economic Review 4-6(44), 928-942.

Garfinkel, I. \& Manski, C. (1992), Evaluating Welfare and Training Programs, Cambrodge and London : Harvard University Press.

Gilbert, L., Kamionka, T. \& Lacroix, G. (2001), 'Les effets des dispositifs publics d'insertion en emploi destinés aux jeunes hommes défavorisés au Québec', Économie et Statistique 345(5), 55-83.

Glaude, M. (1999), L'égalité entre femmes et hommes : où en sommes nous?, in Conseil d'Analyse Economique, ed., 'Égalité entre femmes et hommes : aspects économiques', Vol. 15, La documentation française, pp. 71-103.

Gouriéroux, C. \& Monfort, A. (1996), Simulation-Based Econometric Methods, Core Lectures : Oxford Universitry Press. 
Gritz, M. (1993), 'The Impact of Training on the Frequency and Duration of Employment', Journal of Econometrics 57(1-3), 21-51.

Hajivassiliou, V. \& McFadden, D. (1998), 'The Method of Simulated Scores for the Estimation of LDV Models', Econometrica 66(4), 863-896.

Heckman, J. (1981a), Heterogeneity and State Dependence, in S. Rosen, ed., 'Studies in Labor Markets', Chicago : University of Chicago Press, chapter 3, pp. 91-139.

Heckman, J. (1981b), Statistical Models for Discrete Panel Data, in C. Manski \& D. McFadden, eds, 'Structural Analysis of Discrete Data with Econometric Applications', MIT Press : Cambridge.

Heckman, J., Ichimura, H. \& Smith, J. (1998), 'Characterizing Selection Bias Using Experimental Data', Econometrica 66(5), 1017-1098.

Jones, S. \& Riddell, C. (1999), 'The Measurement of Unemployment : An Empirical Approach', Econometrica 67(1), 147-162.

Lalonde, R. (1995), 'The Promise of Public Sector-Sponsored Training Programs', Journal of Economic Perspectives 2(9), 149-168.

Laroque, G. \& Salanié, B. (1993), 'Simulation-Based Estimation of Models with Lagged Latent Variables', Journal of Applied Econometrics 8(December), S119-S133. Issue Supplement : Special Issue on Econometric Inference Using Simulations Techniques.

Lee, L. (1992), 'On Efficiency of Methods of Simulated Moments and Maximum Simulated Likelihood Estimation of Discrete Response Models', Econometric Theory 8(4), 518-552.

Lerman, S. \& Manski, C. (1981), On the Use of Simulated Frequencies to Approximate Choice Probabilities, in C. Manski \& D. McFadden, eds, 'Structural Analysis of Discrete Data with Econometric Applications', MIT Press : Cambridge, Massachusetts, pp. 305-319.

Magnac, T. (1997), 'Les stages et l'insertion professionnelle des jeunes : une évaluation statistique', Economie et Statistique 304-305(4/5), 75-94.

Magnac, T. (2000a), 'L'apport de la microéconométrie à l'évaluation des politiques publiques', Cahiers d'économie et sociologie rurale 54, 90-113.

Magnac, T. (2000b), 'Subsidised Training and Youth Employment : Distinguishing Unobserved Heterogeneity from State Dependence in Labor Market Histories', The Economic Journal 110(466), 805-837.

McFadden, D. (1974), Conditional Logit Analysis of Qualitative Choice Behavior, in P. Zarembka, ed., 'Frontiers of Econometrics', Academic Press, New York, pp. 105-142.

McFadden, D. (1989), 'A Method of Simulated Moments for Estimation of Discrete Response Models Without Numerical Integration', Econometrica 57(5), 995-1026.

McFadden, D. \& Train, K. (2000), 'Mixed MNL Models for Discrete Response', Journal of Applied Econometrics 15(5), 447-470.

Mealli, F., Pudney, S. \& Thomas, J. (1996), 'Training Duration and Post-Training Outcomes : A Duration-Limited Competing Risks Model', The Economic Journal 106(435), 422-433.

Newey, W. \& McFadden, D. (1994), Large Sample Estimation and Hypothesis Testing, in R. Engle \& D. McFadden, eds, 'Handbook of Econometrics', Vol. 4, Amsterdam, London and New York : Elsevier, North-Holland, pp. 2111-2245.

Pakes, A. \& Pollard, D. (1989), 'Simulation and the Asymptotics of Optimization Estimators', Econometrica 57(5), 1027-1057. 
Piore, M. (1978), 'Dualism in the Labor Market : A Response to Uncertainty and Flux : the Case of France', Revue Economique 29(1), 26-48.

Stern, S. (1997), 'Simulation-Based Estimation', Journal of Economic Literature 35(4), 20062039.

Summers, L. \& Clark, K. (1990), The Dynamics of Youth Unemployment, in L. Summers, ed., 'Understanding Unemployment', Cambridge, Mass. : MIT Press., pp. 48-85.

Wooldridge, J. (2002), 'Simple Solutions to the Initial Conditions Problem in Dynamic, Nonlinear Panel Data Models With Unobserved Heterogeneity', CEMMAP Working Paper CWP18/02. 